\title{
TWO-DIMENSIONAL FOURIER COSINE SERIES EXPANSION METHOD FOR PRICING FINANCIAL OPTIONS*
}

\author{
M. J. RUIJTER ${ }^{\dagger}$ AND C. W. OOSTERLEE ${ }^{\ddagger}$
}

\begin{abstract}
The COS method for pricing European and Bermudan options with one underlying asset was developed in [F. Fang and C. W. Oosterlee, SIAM J. Sci. Comput., 31 (2008), pp. 826-848] and [F. Fang and C. W. Oosterlee, Numer. Math., 114 (2009), pp. 27-62]. In this paper, we extend the method to higher dimensions, with a multidimensional asset price process. The algorithm can be applied to, for example, pricing two-color rainbow options but also to pricing under the popular Heston stochastic volatility model. For smooth density functions, the resulting method converges exponentially in the number of terms in the Fourier cosine series summations; otherwise we achieve algebraic convergence. The use of an FFT algorithm, for asset prices modeled by Lévy processes, makes the algorithm highly efficient. We perform extensive numerical experiments.
\end{abstract}

Key words. Fourier cosine expansion method, European and Bermudan options, two-color rainbow options, basket options, Lévy process, Heston dynamics

AMS subject classifications. 65T40, 65T50, 91G60, 60E10, 62P05

DOI. $10.1137 / 120862053$

1. Introduction. In financial markets traders deal in assets and options, like the well-known call and put options. Besides these, many "exotic" options have been defined that have more complex contract details and are not traded at regulated exchanges.

One class of exotic option contracts is called the class of multicolor rainbow options, whose payoff may depend on multiple assets, like on the average or the maximum of asset prices. The value of the option depends on the contract details and on the underlying asset prices.

Computational finance deals with numerical and computational questions regarding efficient option pricing and calibration. Usually, an asset price model is calibrated to liquidly available plain vanilla options (calls and puts) from a regulated exchange. For the valuation of the exotic options other computational methods are typically used. Option pricing techniques can be divided into the categories of Monte Carlo simulation, partial differential equation (PDE) methods, and Fourier-based methods. Often Monte Carlo methods are used to price high-dimensional option contracts. The method presented here can be seen as an alternative (deterministic) pricing technique, which can deal with multiasset option problems of medium-sized dimensionality, meaning two-dimensional (2D) to approximately five-dimensional (5D) integrals. The method we propose for pricing higher-dimensional options is based on the Fourier transform of the transitional density function and is especially suitable for asset price models in the class of Lévy processes.

*Submitted to the journal's Computational Methods in Science and Engineering section January 11, 2012; accepted for publication (in revised form) June 11, 2012; published electronically September 27, 2012.

http://www.siam.org/journals/sisc/34-5/86205.html

${ }^{\dagger}$ CPB Netherlands Bureau for Economic Policy Analysis, Den Haag, The Netherlands. Current address: Centrum Wiskunde \& Informatica, Amsterdam 1098 XG, The Netherlands (m.j.ruijter@ cwi.nl).

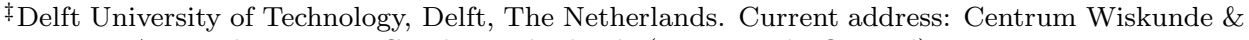
Informatica, Amsterdam 1098 XG, The Netherlands (c.w.oosterlee@cwi.nl).

B642 
In [11], an option pricing method for European options with one underlying asset has been developed, which is called the COS method. The method is based on the Fourier cosine series expansions of the discounted expected payoff. The corresponding characteristic function was used to approximate the Fourier coefficients. For smooth density functions, the error converges exponentially in $N$, the number of terms in the series expansions. The one-dimensional (1D) COS method has been extended in [12] to pricing Bermudan, barrier, and American options. The use of an FFT algorithm, for asset prices modeled by Lévy processes, makes the algorithm highly efficient, with a computational complexity of $O\left(N \log _{2} N\right)$.

The previous strain of literature on the COS method was based on the 1D characteristic function of a single stochastic process. In this paper, we extend the Fourier method to higher dimensions and price in particular two-color rainbow options, which are contracts written on two underlying assets. Well-known examples include the valuation of basket and call-on-maximum options. Methods for both European- and Bermudan-style rainbow options are developed here. The resulting algorithm can be applied to models such as correlated geometric Brownian motions or multidimensional processes with jumps. The method is highly efficient for asset prices in the class of Lévy processes. In the literature, mainly Monte Carlo-based methods are being used to solve higher-dimensional pricing problems; see $[2,3,5,6]$. Leentvaar and Oosterlee worked on a parallel Fourier-based method [22] and parallel sparse grid methods [21] for pricing multiasset options. The authors in [8] demonstrate an implicit PDE discretization method for rainbow options under jump-diffusion processes. We will compare our results to reference values in the literature mentioned above.

The methodology presented here can also be applied to pricing options with one underlying asset, for which the dynamics are governed by two or more correlated stochastic processes. For example, the popular Heston model describes an asset price process with a stochastic volatility [14]. The instantaneous variance process follows a mean-reverting square root (CIR) process. This model is able to capture smile and skew patterns in the implied volatility surface. Besides, the closed-form univariate characteristic function of the log-asset price process makes it easy to implement the Heston model in Fourier-based methods; see [1, 24] for European calls. For the valuation of Bermudan and discrete barrier options, the authors of [13] combined the COS formula for the log-asset dimension and a quadrature rule in the log-variance dimension. Since the bivariate characteristic function of the log-asset price and variance is available, we can also apply the 2D-COS formula to this problem. We investigate the 2D-COS method particularly for Bermudan put options under the Heston dynamics.

The outline of this paper is as follows. We start with the presentation of the 2D-COS formula for pricing European rainbow options (section 2) and the 2D-COS method for solving Bermudan pricing problems (section 3). Section 4 discusses option pricing under the Heston model, which is an affine diffusion process, but not in the Lévy class. The error analysis in section 5 indicates an exponentially converging error for smooth density functions. A nonsmooth density function results in algebraic convergence. Then, in sections 6 and 7, numerical tests are performed. The 2D-COS method can easily be extended to higher dimensions, and we give some insights into the possibilities and difficulties in section 8. Section 9 concludes.

2. European rainbow options. In this section, we explain the $2 D$-COS formula for approximating discounted expected payoffs. The method is based on the Fourier cosine series of the payoff function and the density. The density function of a 
stochastic process is usually not known, but often its characteristic function is known (see $[9,11])$. This enables us to approximate the Fourier coefficients efficiently.

Let $(\Omega, \mathcal{F}, P)$ be a probability space, $T>0$ be a finite terminal time, and $\mathbb{F}=$ $\left(\mathcal{F}_{s}\right)_{0 \leq s \leq T}$ be a filtration satisfying the usual conditions. The process $\mathbf{X}_{t}=\left(X_{t}^{1}, X_{t}^{2}\right)$ denotes a $2 \mathrm{D}$ stochastic process on the filtered probability space $(\Omega, \mathcal{F}, \mathbb{F}, P)$, representing the log-asset prices. We assume that the bivariate characteristic function of the stochastic process is known, which is the case, for example, for affine jumpdiffusions [9]. The value of a European rainbow option, with payoff function $g($.$) , is$ given by the risk-neutral option valuation formula

$$
v\left(t_{0}, \mathbf{x}\right)=e^{-r \Delta t} \mathbb{E}^{t_{0}, \mathbf{x}}\left[g\left(\mathbf{X}_{T}\right)\right]=e^{-r \Delta t} \iint_{\mathbb{R}^{2}} g(\mathbf{y}) f(\mathbf{y} \mid \mathbf{x}) d \mathbf{y} .
$$

Here, $\mathbf{x}=\left(x_{1}, x_{2}\right)$ is the current state, $f\left(y_{1}, y_{2} \mid x_{1}, x_{2}\right)$ is the conditional density function, $r$ is the risk-free rate, and time to expiration is denoted by $\Delta t:=T-t_{0}$. In the derivation of the COS formula, we distinguish three different approximation steps. The errors introduced in each step are discussed in section 5.1.

Step 1. We assume that the integrand is integrable, which is common for the problems we deal with. Because of that, we can, for given $\mathbf{x}$, truncate the infinite integration ranges to some domain $\left[a_{1}, b_{1}\right] \times\left[a_{2}, b_{2}\right] \subset \mathbb{R}^{2}$ without losing significant accuracy. This gives the multidimensional Fourier cosine expansion formulation

$$
\begin{aligned}
v_{1}\left(t_{0}, \mathbf{x}\right)= & e^{-r \Delta t} \int_{a_{2}}^{b_{2}} \int_{a_{1}}^{b_{1}} g(\mathbf{y}) f(\mathbf{y} \mid \mathbf{x}) d y_{1} d y_{2} \\
= & e^{-r \Delta t} \int_{a_{2}}^{b_{2}} \int_{a_{1}}^{b_{1}} g(\mathbf{y}) \sum_{k_{1}=0}^{+\infty} \sum_{k_{2}=0}^{+\infty} A_{k_{1}, k_{2}}(\mathbf{x}) \\
& \cos \left(k_{1} \pi \frac{y_{1}-a_{1}}{b_{1}-a_{1}}\right) \cos \left(k_{2} \pi \frac{y_{2}-a_{2}}{b_{2}-a_{2}}\right) d y_{1} d y_{2} .
\end{aligned}
$$

The notation $v_{i}$ is used for the different approximations of $v$ and keeps track of the numerical errors that set in from each step. For final approximations we also use the "hat" notation, like $\hat{v}, \hat{c}$, etc. In the second line in (2.2), the conditional density is replaced by its Fourier cosine expansion in $\mathbf{y}$ on $\left[a_{1}, b_{1}\right] \times\left[a_{2}, b_{2}\right]$, with series coefficients $A_{k_{1}, k_{2}}(\mathbf{x})$ defined by

$$
A_{k_{1}, k_{2}}(\mathbf{x}):=\frac{2}{b_{1}-a_{1}} \frac{2}{b_{2}-a_{2}} \int_{a_{2}}^{b_{2}} \int_{a_{1}}^{b_{1}} f(\mathbf{y} \mid \mathbf{x}) \cos \left(k_{1} \pi \frac{y_{1}-a_{1}}{b_{1}-a_{1}}\right) \cos \left(k_{2} \pi \frac{y_{2}-a_{2}}{b_{2}-a_{2}}\right) d y_{1} d y_{2} .
$$

$\sum^{\prime}$ in (2.2) means that the first term of the summation has half weight. We interchange summation and integration and define

$$
V_{k_{1}, k_{2}}(T):=\frac{2}{b_{1}-a_{1}} \frac{2}{b_{2}-a_{2}} \int_{a_{2}}^{b_{2}} \int_{a_{1}}^{b_{1}} g(\mathbf{y}) \cos \left(k_{1} \pi \frac{y_{1}-a_{1}}{b_{1}-a_{1}}\right) \cos \left(k_{2} \pi \frac{y_{2}-a_{2}}{b_{2}-a_{2}}\right) d y_{1} d y_{2}
$$

which are the Fourier cosine series coefficients of $v(T, \mathbf{y})=g(\mathbf{y})$ on $\left[a_{1}, b_{1}\right] \times\left[a_{2}, b_{2}\right]$.

Step 2. Truncation of the series summations gives

$$
v_{2}\left(t_{0}, \mathbf{x}\right)=\frac{b_{1}-a_{1}}{2} \frac{b_{2}-a_{2}}{2} e^{-r \Delta t} \sum_{k_{1}=0}^{N_{1}-1} \sum_{k_{2}=0}^{\prime N_{2}-1} A_{k_{1}, k_{2}}(\mathbf{x}) V_{k_{1}, k_{2}}(T) .
$$

Copyright $@$ by SIAM. Unauthorized reproduction of this article is prohibited. 
Step 3. Next, the coefficients $A_{k_{1}, k_{2}}(\mathbf{x})$ are approximated by

$$
F_{k_{1}, k_{2}}(\mathbf{x}):=\frac{2}{b_{1}-a_{1}} \frac{2}{b_{2}-a_{2}} \iint_{\mathbb{R}^{2}} f(\mathbf{y} \mid \mathbf{x}) \cos \left(k_{1} \pi \frac{y_{1}-a_{1}}{b_{1}-a_{1}}\right) \cos \left(k_{2} \pi \frac{y_{2}-a_{2}}{b_{2}-a_{2}}\right) d y_{1} d y_{2} .
$$

The 2D-COS formula is based on the following goniometric relation [20]:

$$
2 \cos (\alpha) \cos (\beta)=\cos (\alpha+\beta)+\cos (\alpha-\beta) .
$$

With this we obtain

$$
2 F_{k_{1}, k_{2}}(\mathbf{x})=F_{k_{1}, k_{2}}^{+}(\mathbf{x})+F_{k_{1}, k_{2}}^{-}(\mathbf{x})
$$

where

$$
F_{k_{1}, k_{2}}^{ \pm}(\mathbf{x}):=\frac{2}{b_{1}-a_{1}} \frac{2}{b_{2}-a_{2}} \iint_{\mathbb{R}^{2}} f(\mathbf{y} \mid \mathbf{x}) \cos \left(k_{1} \pi \frac{y_{1}-a_{1}}{b_{1}-a_{1}} \pm k_{2} \pi \frac{y_{2}-a_{2}}{b_{2}-a_{2}}\right) d y_{1} d y_{2} .
$$

Now, the coefficients $F_{k_{1}, k_{2}}^{ \pm}(\mathbf{x})$ can be calculated by

$$
\begin{aligned}
& F_{k_{1}, k_{2}}^{ \pm}(\mathbf{x}) \\
& =\frac{2}{b_{1}-a_{1}} \frac{2}{b_{2}-a_{2}} \operatorname{Re}\left(\iint_{\mathbb{R}^{2}} f(\mathbf{y} \mid \mathbf{x}) \exp \left(i k_{1} \pi \frac{y_{1}}{b_{1}-a_{1}} \pm i k_{2} \pi \frac{y_{2}}{b_{2}-a_{2}}\right) d \mathbf{y}\right. \\
& \left.\quad \exp \left(-i k_{1} \pi \frac{a_{1}}{b_{1}-a_{1}} \mp i k_{2} \pi \frac{a_{2}}{b_{2}-a_{2}}\right)\right) \\
& =\frac{2}{b_{1}-a_{1}} \frac{2}{b_{2}-a_{2}} \operatorname{Re}\left(\varphi\left(\frac{k_{1} \pi}{b_{1}-a_{1}}, \pm \frac{k_{2} \pi}{b_{2}-a_{2}} \mid \mathbf{x}\right) \exp \left(-i k_{1} \pi \frac{a_{1}}{b_{1}-a_{1}} \mp i k_{2} \pi \frac{a_{2}}{b_{2}-a_{2}}\right)\right) \\
& =\frac{2}{b_{1}-a_{1}} \frac{2}{b_{2}-a_{2}} \operatorname{Re}\left(\varphi_{\text {levy }}\left(\frac{k_{1} \pi}{b_{1}-a_{1}}, \pm \frac{k_{2} \pi}{b_{2}-a_{2}}\right) \exp \left(i k_{1} \pi \frac{x_{1}-a_{1}}{b_{1}-a_{1}} \pm i k_{2} \pi \frac{x_{2}-a_{2}}{b_{2}-a_{2}}\right)\right) .
\end{aligned}
$$

$\operatorname{Re}($.$) denotes taking the real part of the input argument. \varphi(., . \mid \mathbf{x})$ is the bivariate conditional characteristic function of $\mathbf{X}_{T}$, given $\mathbf{X}_{t_{0}}=\mathbf{x}[26]$ :

$$
\varphi(\mathbf{u} \mid \mathbf{x})=\mathbb{E}\left[e^{i \mathbf{u} \cdot \mathbf{X}_{T}} \mid \mathcal{F}_{t_{0}}\right]=\iint_{\mathbb{R}^{2}} e^{i \mathbf{u} \cdot \mathbf{y}} f(\mathbf{y} \mid \mathbf{x}) d \mathbf{y} .
$$

Examples of these characteristic functions can be found in section 6 . The last equality in (2.10) holds particularly for Lévy processes, for which $\varphi_{\text {levy }}\left(u_{1}, u_{2}\right):=\varphi\left(u_{1}, u_{2} \mid 0,0\right)$. Inserting (2.10) into (2.5) gives us the $2 D$-COS formula for approximation of $v\left(t_{0}, \mathbf{x}\right)$ :

$$
\begin{aligned}
& \hat{v}\left(t_{0}, \mathbf{x}\right):=\frac{b_{1}-a_{1}}{2} \frac{b_{2}-a_{2}}{2} e^{-r \Delta t} \sum_{k_{1}=0}^{N_{1}-1} \sum_{k_{2}=0}^{N_{2}-1} \frac{1}{2}\left[F_{k_{1}, k_{2}}^{+}(\mathbf{x})+F_{k_{1}, k_{2}}^{-}(\mathbf{x})\right] V_{k_{1}, k_{2}}(T) \\
& =e^{-r \Delta t} \sum_{k_{1}=0}^{N_{1}-1} \sum_{k_{2}=0}^{N_{2}-1} \frac{1}{2}\left[\operatorname { R e } \left(\varphi_{\text {levy }}\left(\frac{k_{1} \pi}{b_{1}-a_{1}},+\frac{k_{2} \pi}{b_{2}-a_{2}}\right)\right.\right. \\
& \left.\exp \left(i k_{1} \pi \frac{x_{1}-a_{1}}{b_{1}-a_{1}}+i k_{2} \pi \frac{x_{2}-a_{2}}{b_{2}-a_{2}}\right)\right) \\
& +\operatorname{Re}\left(\varphi_{\text {levy }}\left(\frac{k_{1} \pi}{b_{1}-a_{1}},-\frac{k_{2} \pi}{b_{2}-a_{2}}\right)\right. \\
& \left.\left.\exp \left(i k_{1} \pi \frac{x_{1}-a_{1}}{b_{1}-a_{1}}-i k_{2} \pi \frac{x_{2}-a_{2}}{b_{2}-a_{2}}\right)\right)\right] V_{k_{1}, k_{2}}(T) .
\end{aligned}
$$

Copyright $@$ by SIAM. Unauthorized reproduction of this article is prohibited. 
With the multidimensional-COS formula, calculation of the option's Greeks is straightforward, as explained for the 1D case in [11].

Remark 1. Cosine terms facilitate the usage of the characteristic function. Fourier sine expansions may also be used; however, their coefficients decrease at a lower rate for the payoff functions discussed, and because of this the cosine series are preferred. Alternative basis functions, like certain wavelet basis functions, may represent another interesting research direction for option pricing, but this is not yet known and is part of future research.

If the characteristic function is not available directly or not known analytically, it may be approximated. Local volatility models, for example, typically do not yield analytic functions $\varphi$, but recent research in [25] proposes a second-order approximation formula, so that an approximate characteristic function may be derived.

3. Bermudan rainbow options. We generalize the multidimensional-COS method to pricing Bermudan rainbow options with a $2 \mathrm{D}$ underlying log-asset price process, $\mathbf{X}_{t}=\left(X_{t}^{1}, X_{t}^{2}\right)$, that is in the class of Lévy processes. A Bermudan option can be exercised at a fixed set of $\mathcal{M}$ early-exercise times $t_{0}<t_{1}<\cdots t_{m}<\cdots<t_{\mathcal{M}}=T$, with $\Delta t:=t_{m+1}-t_{m}$. The payoff function is denoted by $g($.$) . The problem is solved$ backwards in time, with

$$
\left\{\begin{aligned}
v\left(t_{\mathcal{M}}, \mathbf{x}\right) & =g(\mathbf{x}) \\
c\left(t_{m-1}, \mathbf{x}\right) & =e^{-r \Delta t} \mathbb{E}\left[v\left(t_{m}, \mathbf{X}_{t_{m}}\right) \mid \mathbf{X}_{t_{m-1}}=\mathbf{x}\right] \\
v\left(t_{m-1}, \mathbf{x}\right) & =\max \left[g(\mathbf{x}), c\left(t_{m-1}, \mathbf{x}\right)\right], \quad 2 \leq m \leq \mathcal{M} \\
v\left(t_{0}, \mathbf{x}_{0}\right) & =c\left(t_{0}, \mathbf{x}_{0}\right) .
\end{aligned}\right.
$$

Function $c\left(t_{m-1}, \mathbf{x}\right)$ is called the continuation value and is approximated by the 2DCOS formula

$$
\hat{c}\left(t_{m-1}, \mathbf{x}\right):=\frac{b_{1}-a_{1}}{2} \frac{b_{2}-a_{2}}{2} e^{-r \Delta t} \sum_{k_{1}=0}^{N_{1}-1} \sum_{k_{2}=0}^{\prime N_{2}-1} \frac{1}{2}\left[F_{k_{1}, k_{2}}^{+}(\mathbf{x})+F_{k_{1}, k_{2}}^{-}(\mathbf{x})\right] V_{k_{1}, k_{2}}\left(t_{m}\right) .
$$

The Fourier coefficients of the value function in (3.1) are given by

$$
V_{k_{1}, k_{2}}\left(t_{m}\right):=\frac{2}{b_{1}-a_{1}} \frac{2}{b_{2}-a_{2}} \int_{a_{2}}^{b_{2}} \int_{a_{1}}^{b_{1}} v\left(t_{m}, \mathbf{y}\right) \cos \left(k_{1} \pi \frac{y_{1}-a_{1}}{b_{1}-a_{1}}\right) \cos \left(k_{2} \pi \frac{y_{2}-a_{2}}{b_{2}-a_{2}}\right) d y_{1} d y_{2} .
$$

The option function is now approximated by $\hat{v}\left(t_{m-1}, \mathbf{x}\right):=\max \left[g(\mathbf{x}), \hat{c}\left(t_{m-1}, \mathbf{x}\right)\right]$.

3.1. Recursion formula for coefficients $V_{k_{1}, k_{2}}\left(t_{m}\right)$. In this section, a recursive algorithm for recovering the coefficients $V_{k_{1}, k_{2}}\left(t_{m}\right)$, backwards in time, is derived.

In the coefficients $V_{k_{1}, k_{2}}\left(t_{\mathcal{M}}\right)$, the terminal condition $v\left(t_{\mathcal{M}}, \mathbf{y}\right)=g(\mathbf{y})$ appears. Some payoff functions provide analytic solutions to these coefficients in (3.2); otherwise they can be approximated, as explained in section 3.2.1.

For the coefficients that are used to approximate the continuation values at times $t_{0}, \ldots, t_{M-2}$, the value function, $v\left(t_{m}, \mathbf{y}\right)=\max \left[g(\mathbf{y}), c\left(t_{m}, \mathbf{y}\right)\right]$, appears in the terms $V_{k_{1}, k_{2}}\left(t_{m}\right)$ and we need to find an optimal policy for all state values $\mathbf{y} \in\left[a_{1}, b_{1}\right] \times$ $\left[a_{2}, b_{2}\right]$. We divide the domain $\left[a_{1}, b_{1}\right] \times\left[a_{2}, b_{2}\right]$ into rectangular subdomains $\mathcal{C}^{q}$ and $\mathcal{G}^{p}$, so that approximately for all states $\mathbf{y} \in \mathcal{C}^{q}$ it is optimal to continue and for all $\mathbf{y} \in \mathcal{G}^{p}$ it is optimal to exercise the option. The concept is demonstrated in Figure 3.1 for a call-on-maximum option. The blue rectangles represent the earlyexercise regions $\mathcal{G}^{p}$, the green ones are the continuation regions $\mathcal{C}^{q}$, and the dashed 


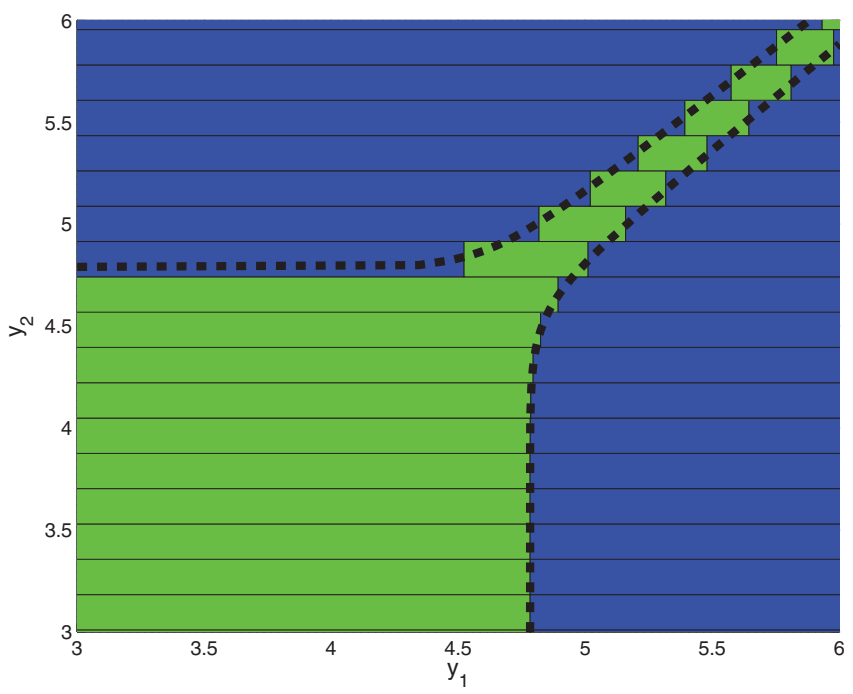

FIG. 3.1. Rectangular regions $\mathcal{C}^{q}$ (green) and $\mathcal{G}^{p}$ (blue).

line shows an accurate boundary. We can split the integral in the definition of $V_{k_{1}, k_{2}}$ into different parts:

$$
\begin{aligned}
V_{k_{1}, k_{2}}\left(t_{m}\right) & =\frac{2}{b_{1}-a_{1}} \frac{2}{b_{2}-a_{2}} \sum_{p} \iint_{\mathcal{G}^{p}} g(\mathbf{y}) \cos \left(k_{1} \pi \frac{y_{1}-a_{1}}{b_{1}-a_{1}}\right) \cos \left(k_{2} \pi \frac{y_{2}-a_{2}}{b_{2}-a_{2}}\right) d \mathbf{y} \\
& +\frac{2}{b_{1}-a_{1}} \frac{2}{b_{2}-a_{2}} \sum_{q} \iint_{\mathcal{C}^{q}} c\left(t_{m}, \mathbf{y}\right) \cos \left(k_{1} \pi \frac{y_{1}-a_{1}}{b_{1}-a_{1}}\right) \cos \left(k_{2} \pi \frac{y_{2}-a_{2}}{b_{2}-a_{2}}\right) d \mathbf{y} \\
& :=\sum_{p} G_{k_{1}, k_{2}}\left(\mathcal{G}^{p}\right)+\sum_{q} C_{k_{1}, k_{2}}\left(t_{m}, \mathcal{C}^{q}\right) \quad(m \neq 0, \mathcal{M}) .
\end{aligned}
$$

We approximate the terms $C_{k_{1}, k_{2}}\left(t_{\mathcal{M}-1},\left[z_{q}, z_{q+1}\right] \times\left[w_{q}, w_{q+1}\right]\right)$ in (3.3), where the variables $z_{q}, z_{q+1}, w_{q}$, and $w_{q+1}$ denote the corner points of the rectangular continuation region $\mathcal{C}^{q}$. For the integrand of the terms $C_{k_{1}, k_{2}}$ we again apply the 2D Fourier cosine expansion by inserting the COS formula for $c\left(t_{\mathcal{M}-1}, \mathbf{y}\right)$, i.e., (3.1). The approximation reads as

$$
\begin{aligned}
\hat{C}_{k_{1}, k_{2}}\left(t_{\mathcal{M}-1},\left[z_{q}, z_{q+1}\right] \times\left[w_{q}, w_{q+1}\right]\right) \\
:=\frac{2}{b_{1}-a_{1}} \frac{2}{b_{2}-a_{2}} \int_{w_{q}}^{w_{q+1}} \int_{z_{q}}^{z_{q+1}} \hat{c}\left(t_{\mathcal{M}-1}, \mathbf{y}\right) \cos \left(k_{1} \pi \frac{y_{1}-a_{1}}{b_{1}-a_{1}}\right) \cos \left(k_{2} \pi \frac{y_{2}-a_{2}}{b_{2}-a_{2}}\right) d y_{1} d y_{2} \\
=\int_{w_{q}}^{w_{q+1}} \int_{z_{q}}^{z_{q+1}} \sum_{j_{1}=0}^{N_{1}-1} \sum_{j_{2}=0}^{\prime N_{2}-1} e^{-r \Delta t} \frac{1}{2}\left[F_{j_{1}, j_{2}}^{+}(\mathbf{y})+F_{j_{1}, j_{2}}^{-}(\mathbf{y})\right] \\
\quad V_{j_{1}, j_{2}}\left(t_{M}\right) \cos \left(k_{1} \pi \frac{y_{1}-a_{1}}{b_{1}-a_{1}}\right) \cos \left(k_{2} \pi \frac{y_{2}-a_{2}}{b_{2}-a_{2}}\right) d y_{1} d y_{2}
\end{aligned}
$$




$$
\begin{aligned}
& =\operatorname{Re}\left(\sum_{j_{1}=0}^{N_{1}-1} \sum_{j_{2}=0}^{N_{2}-1} \frac{1}{2} e^{-r \Delta t} \varphi_{\text {levy }}\left(\frac{j_{1} \pi}{b_{1}-a_{1}},+\frac{j_{2} \pi}{b_{2}-a_{2}}\right)\right. \\
& \left.V_{j_{1}, j_{2}}\left(t_{\mathcal{M}}\right) M_{k_{1}, j_{1}}^{+}\left(z_{q}, z_{q+1}, a_{1}, b_{1}\right) M_{k_{2}, j_{2}}^{+}\left(w_{q}, w_{q+1}, a_{2}, b_{2}\right)\right) \\
& +\operatorname{Re}\left(\sum_{j_{1}=0}^{N_{1}-1} \sum_{j_{2}=0}^{N_{2}-1} \frac{1}{2} e^{-r \Delta t} \varphi_{\text {levy }}\left(\frac{j_{1} \pi}{b_{1}-a_{1}},-\frac{j_{2} \pi}{b_{2}-a_{2}}\right)\right. \\
& \left.V_{j_{1}, j_{2}}\left(t_{\mathcal{M}}\right) M_{k_{1}, j_{1}}^{+}\left(z_{q}, z_{q+1}, a_{1}, b_{1}\right) M_{k_{2}, j_{2}}^{-}\left(w_{q}, w_{q+1}, a_{2}, b_{2}\right)\right),
\end{aligned}
$$

where the elements of square-matrices $M^{+}$and $M^{-}$are given by

$$
\begin{aligned}
& M_{m, n}^{+}\left(u_{1}, u_{2}, a, b\right):=\frac{2}{b-a} \int_{u_{1}}^{u_{2}} e^{i n \pi \frac{y-a}{b-a}} \cos \left(m \pi \frac{y-a}{b-a}\right) d y, \\
& M_{m, n}^{-}\left(u_{1}, u_{2}, a, b\right):=\frac{2}{b-a} \int_{u_{1}}^{u_{2}} e^{-i n \pi \frac{y-a}{b-a}} \cos \left(m \pi \frac{y-a}{b-a}\right) d y .
\end{aligned}
$$

We thus find

$$
\hat{C}_{k_{1}, k_{2}}\left(t_{\mathcal{M}-1},\left[z_{q}, z_{q+1}\right] \times\left[w_{q}, w_{q+1}\right]\right)=\operatorname{Re}\left(\sum_{j_{1}=0}^{N_{1}-1} M_{k_{1}, j_{1}}^{+}\left(z_{q}, z_{q+1}, a_{1}, b_{1}\right) \mathcal{A}_{j_{1}, k_{2}}^{q}\right),
$$

where

$$
\begin{aligned}
\mathcal{A}_{j_{1}, k_{2}}^{q} & :=\sum_{j_{2}=0}^{N_{2}-1} \frac{1}{2} e^{-r \Delta t} \varphi_{\text {levy }}\left(\frac{j_{1} \pi}{b_{1}-a_{1}},+\frac{j_{2} \pi}{b_{2}-a_{2}}\right) V_{j_{1}, j_{2}}\left(t_{\mathcal{M}}\right) M_{k_{2}, j_{2}}^{+}\left(w_{q}, w_{q+1}, a_{2}, b_{2}\right) \\
& +\sum_{j_{2}=0}^{N_{2}-1} \frac{1}{2} e^{-r \Delta t} \varphi_{\text {levy }}\left(\frac{j_{1} \pi}{b_{1}-a_{1}},-\frac{j_{2} \pi}{b_{2}-a_{2}}\right) V_{j_{1}, j_{2}}\left(t_{\mathcal{M}}\right) M_{k_{2}, j_{2}}^{-}\left(w_{q}, w_{q+1}, a_{2}, b_{2}\right) .
\end{aligned}
$$

The elements of $\left(N_{1} \times N_{2}\right)$-matrix $\mathcal{A}^{q}$ are calculated in a rowwise fashion. The rowvector $\mathcal{A}_{j_{1}, .}^{q}=\left\{\mathcal{A}_{j_{1}, k_{2}}^{q}\right\}_{k_{2}=0}^{N_{2}-1}$ can be written as two matrix-vector multiplications:

$$
\mathcal{A}_{j_{1}, .}^{q}=M^{+}\left(w_{q}, w_{q+1}, a_{2}, b_{2}\right) \mathbf{w}_{j_{1}, .}^{q+}+M^{-}\left(w_{q}, w_{q+1}, a_{2}, b_{2}\right) \mathbf{w}_{j_{1},}^{q-},
$$

where

$$
\mathbf{w}_{j_{1}, .}^{q \pm}:=\left\{w_{j_{1}, j_{2}}^{q \pm}\right\}_{j_{2}=0}^{N_{2}-1}, \quad \text { with } \quad w_{j_{1}, j_{2}}^{q \pm}:=\frac{1}{2} e^{-r \Delta t} \varphi_{\text {levy }}\left(\frac{j_{1} \pi}{b_{1}-a_{1}}, \pm \frac{j_{2} \pi}{b_{2}-a_{2}}\right) V_{j_{1}, j_{2}}\left(t_{\mathcal{M}}\right) .
$$

Then, the matrix $\hat{C}_{k_{1}, k_{2}}$ is computed in a columnwise fashion. The column-vector $\hat{C}_{., k_{2}}=\left\{\hat{C}_{k_{1}, k_{2}}\right\}_{k_{1}=0}^{N_{1}-1}$ is calculated by one matrix-vector product,

$$
\hat{C}_{., k_{2}}\left(t_{\mathcal{M}-1},\left[z_{q}, z_{q+1}\right] \times\left[w_{q}, w_{q+1}\right]\right)=\operatorname{Re}\left(M^{+}\left(z_{q}, z_{q+1}, a_{1}, b_{1}\right) \mathcal{A}_{., k_{2}}^{q}\right),
$$

with column-vector $\mathcal{A}_{., k_{2}}^{q}=\left\{\mathcal{A}_{j_{1}, k_{2}}^{q}\right\}_{j_{1}=0}^{N_{1}-1}$.

Copyright $@$ by SIAM. Unauthorized reproduction of this article is prohibited. 
The coefficients $G_{k_{1}, k_{2}}\left(\left[z_{p}, z_{p+1}\right] \times\left[w_{p}, w_{p+1}\right]\right)$ are defined by

$$
\begin{aligned}
& G_{k_{1}, k_{2}}\left(\left[z_{p}, z_{p+1}\right] \times\left[w_{p}, w_{p+1}\right]\right) \\
& \quad=\frac{2}{b_{1}-a_{1}} \frac{2}{b_{2}-a_{2}} \int_{w_{p}}^{w_{p+1}} \int_{z_{p}}^{z_{p+1}} g(\mathbf{y}) \cos \left(k_{1} \pi \frac{y_{1}-a_{1}}{b_{1}-a_{1}}\right) \cos \left(k_{2} \pi \frac{y_{2}-a_{2}}{b_{2}-a_{2}}\right) d y_{1} d y_{2} .
\end{aligned}
$$

These terms may admit an analytic solution; however, in some practical applications an analytic solution is not present. Methods to approximate these terms are proposed in section 3.2.2.

We end up with the approximated coefficients

$$
\hat{V}_{k_{1}, k_{2}}\left(t_{\mathcal{M}-1}\right):=\sum_{p} G_{k_{1}, k_{2}}\left(\mathcal{G}^{p}\right)+\sum_{q} \hat{C}_{k_{1}, k_{2}}\left(t_{\mathcal{M}-1}, \mathcal{C}^{q}\right) .
$$

For the other coefficients $V_{k_{1}, k_{2}}\left(t_{m}\right)$, the approximations $\hat{c}\left(t_{m}, \mathbf{y}\right)$ and $\hat{V}_{j_{1}, j_{2}}\left(t_{m+1}\right)$ will be used to approximate the terms $C_{k_{1}, k_{2}}\left(t_{m},\left[z_{q}, z_{q+1}\right] \times\left[w_{q}, w_{q+1}\right]\right)$, and the elements of the corresponding matrix $\mathcal{A}^{q}$ are

$$
\begin{aligned}
\mathcal{A}_{j_{1}, k_{2}}^{q} & =\sum_{j_{2}=0}^{N_{2}-1} \frac{1}{2} e^{-r \Delta t} \varphi_{\text {levy }}\left(\frac{j_{1} \pi}{b_{1}-a_{1}},+\frac{j_{2} \pi}{b_{2}-a_{2}}\right) \hat{V}_{j_{1}, j_{2}}\left(t_{m+1}\right) M_{k_{2}, j_{2}}^{+}\left(w_{q}, w_{q+1}, a_{2}, b_{2}\right) \\
& +\sum_{j_{2}=0}^{N_{2}-1} \frac{1}{2} e^{-r \Delta t} \varphi_{\text {levy }}\left(\frac{j_{1} \pi}{b_{1}-a_{1}},-\frac{j_{2} \pi}{b_{2}-a_{2}}\right) \hat{V}_{j_{1}, j_{2}}\left(t_{m+1}\right) M_{k_{2}, j_{2}}^{-}\left(w_{q}, w_{q+1}, a_{2}, b_{2}\right) .
\end{aligned}
$$

FFT. The matrix-vector products $M^{+} \mathbf{v}$ and $M^{-} \mathbf{v}$ in the computation of matrices $\mathcal{A}^{q}$ and $\hat{C}$ can be computed efficiently by a Fourier-based algorithm, as stated in Theorem A.1 (Appendix A). The computation time achieved is $O\left(N \log _{2} N\right)$, with $N$ the length of the vector.

Algorithm. We can recover the terms $\hat{V}_{k_{1}, k_{2}}\left(t_{m}\right)$ recursively, starting with $V_{k_{1}, k_{2}}\left(t_{\mathcal{M}}\right)$. The algorithm for solving the pricing problem backwards in time reads as follows.

\section{Algorithm 1. (2D-COS method for pricing Bermudan rainbow op- tions) \\ Initialization: Calculate coefficients $V_{k_{1}, k_{2}}\left(t_{\mathcal{M}}\right)$. \\ Main loop to recover $\hat{V}\left(t_{m}\right)$ : \\ For $m=\mathcal{M}-1$ to 1 : \\ - Determine the optimal continuation regions $\mathcal{C}^{q}$ and early- exercise regions $\mathcal{G}^{p}$, as in Figure 3.1. \\ - Compute $\hat{V}\left(t_{m}\right)$ from (3.3) with the help of the FFT algorithm.}

Final step: Compute $\hat{v}\left(t_{0}, \mathbf{x}_{0}\right)$ by inserting $\hat{V}_{k_{1}, k_{2}}\left(t_{1}\right)$ into (3.1).

Computational complexity. The initialization is of order $O\left(N_{1} N_{2}\right)$. In the main loop there are $\mathcal{M}-1$ iterations in which the following computations are performed. The construction of one matrix $\mathcal{A}^{q}$ costs $O\left(2 N_{1} N_{2} \log _{2} N_{2}\right)$ operations. Computation of $\hat{C}_{k_{1}, k_{2}}\left(t_{m},\left[z_{q}, z_{q+1}\right] \times\left[w_{q}, w_{q+1}\right]\right)$ takes $O\left(N_{2} N_{1} \log _{2} N_{1}\right)$ operations. $G_{k_{1}, k_{2}}\left(\left[z_{p}, z_{p+1}\right] \times\left[w_{p}, w_{p+1}\right]\right)$ is of order $O\left(N_{1} N_{2}\right)$. The computation time is linear in the number of continuation and early-exercise regions. The final step takes $O\left(N_{1} N_{2}\right)$ operations. 
3.2. Approximation methods for the coefficients $V(T)$ and $G\left(\mathcal{G}^{p}\right)$. In this section, we propose methods for approximating the terminal coefficients $V_{k_{1}, k_{2}, \ldots, k_{n}}(T)$ and the terms $G_{k_{1}, k_{2}, \ldots, k_{n}}\left(\mathcal{G}^{p}\right)$ that are specific for the multidimensional-COS method.

In the $1 \mathrm{D}$ pricing problem, the terminal coefficients $V_{k_{1}}(T)$ admit analytic solutions for several options, like put- and call-based options, digital options, and power options. Besides, in the 1D-COS method for pricing Bermudan options, the terms $G_{k_{1}}\left(\mathcal{G}^{p}\right)$ are also usually known analytically.

In two dimensions, the payoff functions of, for instance, a geometric basket or a call-on-maximum option provide analytic solutions to the 2D coefficients $V_{k_{1}, k_{2}}(T)$, but this is generally an exception. If no exact representation is available, then they can be approximated by using discrete cosine transforms (DCTs) or the ClenshawCurtis quadrature rule. The usage of DCTs is explained in section 3.2.1. Also, analytic forms for the terms $G_{k_{1}, k_{2}, \ldots, k_{n}}\left(\mathcal{G}^{p}\right)$ are in general not available in the multidimensional version. An approximation method, based on Fourier cosine expansion of the payoff function, is discussed in section 3.2.2.

3.2.1. DCTs. In this section, we explain this idea of using DCTs to approximate the terminal coefficients $V_{k_{1}, k_{2}}(T)$. For this, we take $Q \geq \max \left[N_{1}, N_{2}\right]$ grid-points for each spatial dimension and define

$$
y_{i}^{n_{i}}:=a_{i}+\left(n_{i}+\frac{1}{2}\right) \frac{b_{i}-a_{i}}{Q} \text { and } \Delta y_{i}:=\frac{b_{i}-a_{i}}{Q}, \quad i=1,2 .
$$

The midpoint-rule integration gives us

$$
\begin{aligned}
V_{k_{1}, k_{2}}(T) \approx & \sum_{n_{1}=0}^{Q-1} \sum_{n_{2}=0}^{Q-1} \frac{2}{b_{1}-a_{1}} \frac{2}{b_{2}-a_{2}} g\left(y_{1}^{n_{1}}, y_{2}^{n_{2}}\right) \cos \left(k_{1} \pi \frac{y_{1}^{n_{1}}-a_{1}}{b_{1}-a_{1}}\right) \\
& \cos \left(k_{2} \pi \frac{y_{2}^{n_{2}}-a_{2}}{b_{2}-a_{2}}\right) \Delta y_{1} \Delta y_{2} \\
= & \sum_{n_{1}=0}^{Q-1} \sum_{n_{2}=0}^{Q-1} g\left(y_{1}^{n_{1}}, y_{2}^{n_{2}}\right) \cos \left(k_{1} \pi \frac{2 n_{1}+1}{2 Q}\right) \cos \left(k_{2} \pi \frac{2 n_{2}+1}{2 Q}\right) \frac{2}{Q} \frac{2}{Q}
\end{aligned}
$$

The above 2D DCT (Type II) can be calculated efficiently by, for example, the function det2 of MATLAB. The approximated coefficients are denoted by $V_{k_{1}, k_{2}}^{D C T}(T)$, with the corresponding computed European option value $\hat{v}^{D C T}\left(t_{0}, \mathbf{x}\right)$. Now, an extra error is introduced:

$$
\begin{aligned}
\epsilon_{D C T}\left(t_{0}, \mathbf{x}\right) & :=\hat{v}\left(t_{0}, \mathbf{x}\right)-\hat{v}^{D C T}\left(t_{0}, \mathbf{x}\right) \\
& =\frac{b_{1}-a_{1}}{2} \frac{b_{2}-a_{2}}{2} e^{-r \Delta t} \sum_{k_{1}=0}^{N_{1}-1} \sum_{k_{2}=0}^{\prime} F_{N_{2}-1}^{\prime} F_{k_{1}, k_{2}}(\mathbf{x})\left[V_{k_{1}, k_{2}}(T)-V_{k_{1}, k_{2}}^{D C T}(T)\right] .
\end{aligned}
$$

This error $\epsilon_{D C T}$ converges algebraically in $Q$, with order two. We will confirm this by an example in section 6.1.1. The DCT method can be extended to higher dimensions for the approximation of coefficients $V_{k_{1}, k_{2}, \ldots, k_{n}}(T)$.

Remark 2. The above approximation, (3.16), is based on the midpoint-rule integration. Higher-order methods or adaptive quadrature rules may improve the efficiency. 
3.2.2. Approximation methods for $G\left(\mathcal{G}^{p}\right)$. The terms $G_{k_{1}, k_{2}}$ are defined by

$$
\begin{aligned}
& G_{k_{1}, k_{2}}\left(\left[z_{p}, z_{p+1}\right] \times\left[w_{p}, w_{p+1}\right]\right) \\
& \quad=\frac{2}{b_{1}-a_{1}} \frac{2}{b_{2}-a_{2}} \int_{w_{p}}^{w_{p+1}} \int_{z_{p}}^{z_{p+1}} g(\mathbf{y}) \cos \left(k_{1} \pi \frac{y_{1}-a_{1}}{b_{1}-a_{1}}\right) \cos \left(k_{2} \pi \frac{y_{2}-a_{2}}{b_{2}-a_{2}}\right) d y_{1} d y_{2} .
\end{aligned}
$$

These terms may admit an analytic solution; however, in many practical applications the calculation of coefficients $G_{k_{1}, k_{2}}\left(\mathcal{G}^{p}\right)$ is time consuming, or an analytic solution is not present. Then, we can use discrete Fourier transforms to approximate them, similarly as in section 3.2.1. Another way is the usage of the Fourier cosine expansion of the payoff function. First, we explain this idea in one dimension.

The COS method for 1D Bermudan options was developed in [12]. For a put option it follows that

$$
V_{k_{1}}\left(t_{m}\right)=G_{k_{1}}(\mathcal{G})+C_{k_{1}}\left(t_{m}, \mathcal{C}\right) .
$$

The coefficients $C_{k_{1}}$ are approximated by

$$
\hat{C}_{k_{1}}\left(t_{m},\left[z_{1}, z_{2}\right]\right)=\operatorname{Re}\left(\sum_{j=0}^{N_{1}-1} e^{-r \Delta t} \varphi_{\text {levy }}\left(\frac{j \pi}{b-a}\right) V_{j}\left(t_{m+1}\right) M_{k_{1}, j_{1}}^{+}\left(z_{1}, z_{2}, a, b\right)\right) .
$$

In [12], the coefficients $G_{k_{1}}\left(z_{1}, z_{2}\right)$ are assumed to be known analytically, and

$$
\hat{V}_{k_{1}}\left(t_{m}\right):=G_{k_{1}}(\mathcal{G})+\hat{C}_{k_{1}}\left(t_{m}, \mathcal{C}\right) .
$$

However, the coefficients $G_{k_{1}}$ can also be approximated by a Fourier series expansion of the payoff function, i.e.,

$$
g(x) \approx \hat{g}(x)=\sum_{k_{1}=0}^{N_{1}-1} \cos \left(k \pi \frac{x-a}{b-a}\right) V_{k_{1}}(T)=\sum_{k_{1}=0}^{N_{1}-1} \operatorname{Re}\left(\exp \left(i k \pi \frac{x-a}{b-a}\right)\right) V_{k_{1}}(T),
$$

with $\left\{V_{k_{1}}(T)\right\}_{k_{1}=0}^{+\infty}$ the terminal coefficients and with $V(T, y)=g(y)$. Inserting function $\hat{g}$ into the terms $G_{k_{1}}$ gives

$$
\hat{G}_{k_{1}}\left(\left[z_{1}, z_{2}\right]\right):=\frac{2}{b-a} \int_{z_{1}}^{z_{2}} \hat{g}(y) \cos \left(k \pi \frac{y-a}{b-a}\right) d y=\operatorname{Re}\left(\sum_{j=0}^{N_{1}-1} V_{j_{1}}(T) M_{k_{1}, j_{1}}^{+}\left(z_{1}, z_{2}, a, b\right)\right) .
$$

The computation of $\hat{C}$ and $\hat{G}$ can now be done simultaneously as

$$
\begin{aligned}
& \hat{V}_{k_{1}}\left(t_{m}\right):=\hat{G}_{k_{1}}(\mathcal{G})+\hat{C}_{k_{1}}\left(t_{m}, \mathcal{C}\right) \\
&= \hat{V}_{k_{1}}(T)-\hat{G}_{k_{1}}(\mathcal{C})+\hat{C}_{k_{1}}\left(t_{m}, \mathcal{C}\right) \\
&= \hat{V}_{k_{1}}(T)+\operatorname{Re}\left(\sum_{j=0}^{N_{1}-1}\left[-V_{j_{1}}(T)+e^{-r \Delta t} \varphi_{\text {levy }}\left(\frac{j \pi}{b-a}\right) V_{j_{1}}\left(t_{m+1}\right)\right]\right. \\
&\left.M_{k_{1}, j_{1}}^{+}(\mathcal{C}, a, b)\right) .
\end{aligned}
$$

Copyright $\odot$ by SIAM. Unauthorized reproduction of this article is prohibited. 
The error of this new approach converges algebraically as the Fourier series of the payoff function. The new approach is a little bit faster than (3.21) (with analytic $G_{k_{1}}(\mathcal{G})$ ); however, a higher value of $N_{1}$ is needed to reach the same accuracy (see Figure 3.2 and Table 1). However, in multiple dimensions this approach may be beneficial and time efficient.

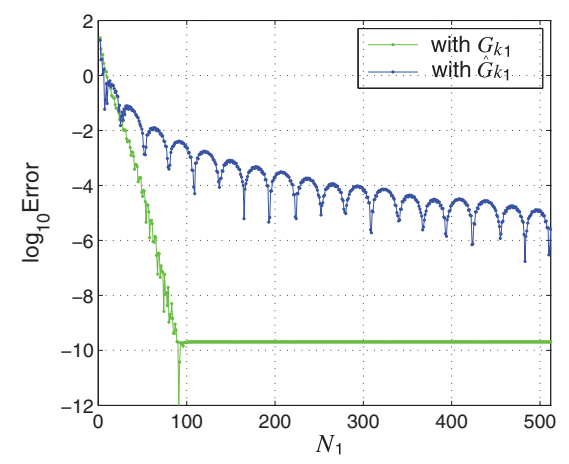

TABLE 1

Error; reference price is 10.479520123 .

\begin{tabular}{r|cc}
\hline$N_{1}$ & Method [12], $(3.21)$ & New method, (3.24) \\
\hline 32 & $-4.893 \mathrm{e}-3$ & $-6.22 \mathrm{e}-2$ \\
64 & $-1.393 \mathrm{e}-6$ & $1.14 \mathrm{e}-2$ \\
128 & $-2.023 \mathrm{e}-10$ & $1.22 \mathrm{e}-3$ \\
256 & $-2.023 \mathrm{e}-10$ & $-4.43 \mathrm{e}-5$ \\
512 & $-2.024 \mathrm{e}-10$ & $2.52 \mathrm{e}-6$ \\
1024 & $-2.024 \mathrm{e}-10$ & $-1.18 \mathrm{e}-7$ \\
\hline
\end{tabular}

Fig. 3.2. Comparison between methods (3.21) and (3.24).

Now we return to (3.18) and explain the approximation method for two dimensions. The Fourier cosine expansion of the payoff function can be written as

$$
\begin{aligned}
\hat{g}(\mathbf{y}) & :=\sum_{k_{1}=0}^{N_{1}-1} \sum_{k_{2}=0}^{\prime} \cos \left(k_{1} \pi \frac{y_{1}-a_{1}}{b_{1}-a_{1}}\right) \cos \left(k_{2} \pi \frac{y_{2}-a_{2}}{b_{2}-a_{2}}\right) V_{k_{1}, k_{2}}(T) \\
& =\sum_{k_{1}=0}^{N_{1}-1} \sum_{k_{2}=0}^{\prime N_{2}-1} \frac{1}{2}\left[\operatorname{Re}\left(\exp \left(i k_{1} \pi \frac{y_{1}-a_{1}}{b_{1}-a_{1}}+i k_{2} \pi \frac{y_{2}-a_{2}}{b_{2}-a_{2}}\right)\right)\right. \\
& \left.+\operatorname{Re}\left(\exp \left(i k_{1} \pi \frac{y_{1}-a_{1}}{b_{1}-a_{1}}-i k_{2} \pi \frac{y_{2}-a_{2}}{b_{2}-a_{2}}\right)\right)\right] V_{k_{1}, k_{2}}(T)
\end{aligned}
$$

With this, the coefficients $G_{k_{1}, k_{2}}$ can by approximated by $\hat{G}_{k_{1}, k_{2}}$, similarly as in (3.4):

$$
\begin{aligned}
& \hat{G}_{k_{1}, k_{2}}\left(\left[z_{p}, z_{p+1}\right] \times\left[w_{p}, w_{p+1}\right]\right) \\
& \quad=\operatorname{Re}\left(\sum_{j_{1}=0}^{N_{1}-1} \sum_{j_{2}=0}^{\prime} \frac{1}{2} V_{j_{1}, j_{2}}(T) M_{k_{1}, j_{1}}^{+}\left(z_{p}, z_{p+1}, a_{1}, b_{1}\right) M_{k_{2}, j_{2}}^{+}\left(w_{p}, w_{p+1}, a_{2}, b_{2}\right)\right) \\
& \quad+\operatorname{Re}\left(\sum_{j_{1}=0}^{N_{1}-1} \sum_{j_{2}=0}^{\prime} \frac{N_{2}-1}{2} V_{j_{1}, j_{2}}(T) M_{k_{1}, j_{1}}^{+}\left(z_{p}, z_{p+1}, a_{1}, b_{1}\right) M_{k_{2}, j_{2}}^{-}\left(w_{p}, w_{p+1}, a_{2}, b_{2}\right)\right)
\end{aligned}
$$

We will use these approximations in sections 6.2 .1 and 6.2.2. This approximation method can be extended to higher dimensions; if the multidimensional coefficients $G_{k_{1}, k_{2}, \ldots, k_{n}}\left(\mathcal{G}^{p}\right)$ are not known analytically, then we can use the terminal coefficients $V_{k_{1}, k_{2}, \ldots, k_{n}}(T)$ to approximate them.

Copyright $\odot$ by SIAM. Unauthorized reproduction of this article is prohibited. 
4. Bermudan options under the Heston model. In this section, we explain the 2D-COS method for a Bermudan pricing problem, in which the asset prices follow the Heston dynamics. This 2D stochastic process is one of the important processes in financial option pricing. In [13], Bermudan options under the Heston stochastic volatility model are priced using the COS formula for the log-asset dimension, combined with a quadrature rule in the log-variance dimension. For this, the closed-form density function of the transformed log-variance process is used. In our approach we employ the bivariate characteristic function of the log-asset price and the variance, which is also available in closed form, and the 2D-COS formula can be applied. However, the 2D stochastic process is not in the class of Lévy processes, and efficient matrix-vector multiplication using the FFT algorithm (Appendix A) within the COS method will therefore be applicable in only one dimension, similarly as in [28].

Let process $\mathbf{X}_{t}=\left(X_{t}^{1}, X_{t}^{2}\right)=\left(X_{t}, \nu_{t}\right)$ represent the Heston model. $X_{t}$ represents the log-asset price process and $\nu_{t}$ is the variance process $\left(\nu_{0} \geq 0\right)$, with dynamics

$$
\begin{aligned}
d X_{t} & =\left(r-\frac{1}{2} \nu_{t}\right) d t+\rho \sqrt{\nu_{t}} d W_{t}^{1}+\sqrt{1-\rho^{2}} \sqrt{\nu_{t}} d W_{t}^{2}, \\
d \nu_{t} & =\kappa\left(\bar{\nu}-\nu_{t}\right) d t+\eta \sqrt{\nu_{t}} d W_{t}^{1} .
\end{aligned}
$$

Here, $r$ represents the risk free rate, $\kappa>0$ the mean reversion rate, $\bar{\nu}>0$ the long run variance, $\eta>0$ the volatility of variance (vol-of-vol), and $\rho$ the correlation coefficient. $\mathbf{W}_{t}$ is a $2 \mathrm{D}$ Brownian motion on the filtered probability space $(\Omega, \mathcal{F}, \mathbb{F}, P)$. The variance process remains strictly positive if the Feller condition, $2 \kappa \bar{\nu} \geq \eta^{2}$, is satisfied; otherwise the boundary at zero is attainable and strongly reflecting [17].

The process $\mathbf{X}_{t}$ is affine, and the bivariate characteristic function is of the form

$$
\begin{aligned}
\phi\left(\mathbf{u}, \mathbf{X}_{t}, t, T\right) & =\mathbb{E}\left[e^{i u_{1} X_{T}+i u_{2} \nu_{T}} \mid X_{t}, \nu_{t}\right] \\
& =\exp \left(B_{1}(T-t, \mathbf{u}) X_{t}+B_{2}(T-t, \mathbf{u}) \nu_{t}+A(T-t, \mathbf{u})\right) .
\end{aligned}
$$

We define

$$
\begin{aligned}
\beta & :=\kappa-i \rho \eta u_{1}, \\
D & :=\sqrt{\beta^{2}+\eta^{2} u_{1}\left(i+u_{1}\right)}, \\
h & :=\left(\beta-D-i u_{2} \eta^{2}\right) /\left(\beta+D-i u_{2} \eta^{2}\right) .
\end{aligned}
$$

The functions $A, B_{1}$, and $B_{2}$ are solutions to a system of ordinary differential equations (ODEs) of Riccati type [9]:

$$
\begin{aligned}
\frac{\partial B_{1}(t, \mathbf{u})}{\partial t} & =0, \\
\frac{\partial B_{2}(t, \mathbf{u})}{\partial t} & =\frac{1}{2} \eta^{2} B_{2}^{2}(t, \mathbf{u})-\beta B_{2}(t, \mathbf{u})-\frac{1}{2} u_{1}\left(i+u_{1}\right), \\
\frac{\partial A(t, \mathbf{u})}{\partial t} & =i u_{1} r+\kappa \bar{\nu} B_{2}(t, \mathbf{u}),
\end{aligned}
$$

with initial conditions $B_{1}(0, \mathbf{u})=i u_{1}, B_{2}(0, \mathbf{u})=i u_{2}, A(0, \mathbf{u})=0$. Solving the ODEs gives

$$
\begin{aligned}
B_{1}(t, \mathbf{u}) & =i u_{1}, \\
B_{2}(t, \mathbf{u}) & =\frac{1}{\eta^{2}} \frac{\beta-D-(\beta+D) h e^{-D t}}{1-h e^{-D t}} \\
A(t, \mathbf{u}) & =i u_{1} r t+\frac{\kappa \bar{\nu}}{\eta^{2}}\left[(\beta-D) t-2 \ln \left(\frac{h e^{-D t}-1}{h-1}\right)\right] .
\end{aligned}
$$

Copyright $@$ by SIAM. Unauthorized reproduction of this article is prohibited. 
Remark 3. The characteristic function involves a multivalued complex logarithm in $A(t, \mathbf{u})$. Most software packages restrict the logarithm to its principal branch. Then, the characteristic function can become discontinuous in $\mathbf{u}$, which results in incorrect option prices. The same problem arises in the 1D characteristic function:

$$
\phi^{1 D}\left(u_{1}, \mathbf{X}_{t}, t, T\right)=\mathbb{E}\left[e^{i u_{1} X_{T}} \mid X_{t}, \nu_{t}\right] .
$$

Solutions to the problem of choosing the correct branch here are discussed extensively in $[23,24,1,19]$. In [24], the function $A\left(t, u_{1}\right)\left(u_{2}=0\right)$ appears in the expression for the 1D characteristic function. They prove that the argument of the logarithm never crosses the negative real axis, so that the principle branch is the correct one. We have not been able to complete the proof that justifies the use of the principle branch for $A(t, \mathbf{u})$ in two dimensions. However, experiments showed that the logarithm's argument does not cross the real negative real axis for the parameter values in section 7 .

The variance process can be expressed as a time-changed squared Bessel process with dimension $\delta:=\frac{4 \kappa \bar{\nu}}{\eta^{2}}$, which has an absolutely continuous distribution if $\delta>0$ (i.e., a density exists) and a probability mass at the origin if $\delta=0[18,10]$. The assumptions on the parameters yield $\delta>0$, which justifies the use of the analytic density function in (2.1) and approximated coefficients $F_{k_{1}, k_{2}}$ (see (2.10)), with characteristic function given by (2.11).

We apply the 2D-COS method to price Bermudan options under the Heston dynamics. We again take fixed time steps $\Delta t:=t_{m+1}-t_{m}$ and define

$$
\begin{aligned}
\varphi\left(u_{1}, u_{2} \mid x, \nu\right) & :=\phi\left(\mathbf{u}, \mathbf{x}, t_{m}, t_{m+1}\right):=e^{i u_{1} x} e^{B_{2}(\Delta t, \mathbf{u}) \nu} \varphi_{A}(\mathbf{u}), \text { where } \\
\varphi_{A}(\mathbf{u}) & :=e^{A(\Delta t, \mathbf{u})} .
\end{aligned}
$$

The approximation of the coefficients $C_{k_{1}, k_{2}}$ now reads as

$$
\begin{aligned}
& \hat{C}_{k_{1}, k_{2}}\left(t_{\mathcal{M}-1},\left[z_{q}, z_{q+1}\right] \times\left[w_{q}, w_{q+1}\right]\right)
\end{aligned}
$$

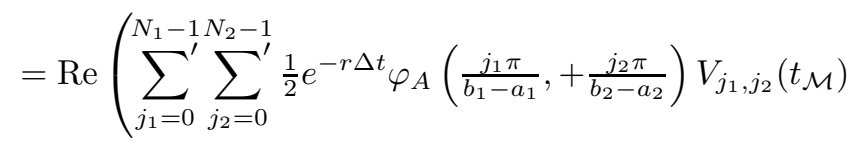

$$
\begin{aligned}
& \left.M_{k_{1}, j_{1}}^{+}\left(z_{q}, z_{q+1}, a_{1}, b_{1}\right) H_{k_{2}, j_{2}}^{+}\left(w_{q}, w_{q+1}, a_{2}, b_{2}, \frac{j_{1} \pi}{b_{1}-a_{1}}\right)\right) \\
& +\operatorname{Re}\left(\sum_{j_{1}=0}^{N_{1}-1} \sum_{j_{2}=0}^{\prime} \frac{N_{2}-1}{2} e^{-r \Delta t} \varphi_{A}\left(\frac{j_{1} \pi}{b_{1}-a_{1}},-\frac{j_{2} \pi}{b_{2}-a_{2}}\right) V_{j_{1}, j_{2}}\left(t_{\mathcal{M}}\right)\right. \\
& \left.M_{k_{1}, j_{1}}^{+}\left(z_{q}, z_{q+1}, a_{1}, b_{1}\right) H_{k_{2}, j_{2}}^{-}\left(w_{q}, w_{q+1}, a_{2}, b_{2}, \frac{j_{1} \pi}{b_{1}-a_{1}}\right)\right) \\
& :=\operatorname{Re}\left(\sum_{j_{1}=0}^{N_{1}-1} M_{k_{1}, j_{1}}^{+}\left(z_{q}, z_{q+1}, a_{1}, b_{1}\right) \mathcal{A}_{j_{1}, k_{2}}^{q}\right),
\end{aligned}
$$

Copyright (c) by SIAM. Unauthorized reproduction of this article is prohibited. 
where the elements of matrices $H^{ \pm}$are given by

$$
\begin{aligned}
& H_{k_{2}, j_{2}}^{ \pm}\left(w_{q}, w_{q+1}, a_{2}, b_{2}, \frac{j_{1} \pi}{b_{1}-a_{1}}\right) \\
& \quad:=\frac{2}{b_{2}-a_{2}} \int_{w_{q}}^{w_{q+1}} e^{y_{2} B_{2}\left(\Delta t, \frac{j_{1} \pi}{b_{1}-a_{1}}, \pm \frac{j_{2} \pi}{b_{2}-a_{2}}\right)} e^{ \pm i j_{2} \pi \frac{-a_{2}}{b_{2}-a_{2}}} \cos \left(k_{2} \pi \frac{y_{2}-a_{2}}{b_{2}-a_{2}}\right) d y_{2} .
\end{aligned}
$$

As before, the elements of $\left(N_{1} \times N_{2}\right)$-matrix $\mathcal{A}^{q}$ are calculated in a rowwise fashion and the row-vector $\mathcal{A}_{j_{1}, .}^{q}=\left\{\mathcal{A}_{j_{1}, k_{2}}^{q}\right\}_{k_{2}=0}^{N_{2}-1}$ can be written as two matrix-vector multiplications,

$$
\mathcal{A}_{j_{1}, .}^{q}=H^{+}\left(w_{q}, w_{q+1}, a_{2}, b_{2}, \frac{j_{1} \pi}{b_{1}-a_{1}}\right) \mathbf{w}_{j_{1}, .}^{q+}+H^{-}\left(w_{q}, w_{q+1}, a_{2}, b_{2}, \frac{j_{1} \pi}{b_{1}-a_{1}}\right) \mathbf{w}_{j_{1},}^{q-},
$$

where

$$
\mathbf{w}_{j_{1}, .}^{q \pm}:=\left\{w_{j_{1}, j_{2}}^{q \pm}\right\}_{j_{2}=0}^{N_{2}-1}, \quad \text { with } \quad w_{j_{1}, j_{2}}^{q \pm}:=\frac{1}{2} e^{-r \Delta t} \varphi_{A}\left(\frac{j_{1} \pi}{b_{1}-a_{1}}, \pm \frac{j_{2} \pi}{b_{2}-a_{2}}\right) V_{j_{1}, j_{2}}\left(t_{\mathcal{M}}\right) .
$$

The above equations are similar to (3.4) and (3.8) for pricing rainbow options. However, the matrices $H^{ \pm}$cannot be decomposed into a Hankel and Toeplitz matrix anymore, and the computation of $\mathcal{A}_{j_{1} \text {,. }}^{q}$ is therefore of order $O\left(N_{2}^{2}\right)$. For the numerical tests in section 7 we will take a fixed grid $\left\{w_{q}\right\}$. Then, the matrices $H^{ \pm}$need to be computed only once, and this computation is part of the algorithm's initialization.

5. Error convergence and computational domain. The method parameters of the 2D-COS method are the integration ranges, $\left[a_{1}, b_{1}\right]$ and $\left[a_{2}, b_{2}\right]$, and the number of Fourier cosine terms, $N_{1}$ and $N_{2}$. Convergence in the number of cosine terms is discussed in the error analysis in section 5.1. Section 5.2 gives some suggestions for the choice of the computational domain.

5.1. Error analysis. The error analysis is similar to the analyses in $[11,12]$. We start with the local error of the COS formula. Then, a result for the propagating error is presented.

The local error of the COS formula is defined by

$$
\epsilon\left(t_{m-1}, \mathbf{x}\right):=c\left(t_{m-1}, \mathbf{x}\right)-\hat{c}\left(t_{m-1}, \mathbf{x}\right) .
$$

Let us assume that the terms $V_{k_{1}, k_{2}}\left(t_{m}\right)$ are known analytically. Then, errors are introduced in three steps (see section 2): the truncation of the integration range, the substitution of the density by its cosine series expansion on the truncated range, and the substitution of the series coefficients by the characteristic function approximation. The key to bounding the error lies in the decay rate of the Fourier cosine series coefficients. We discuss the three errors one after the other.

Step 1. The integration range truncation error:

$\epsilon_{1}\left(t_{m-1}, \mathbf{x}\right):=c\left(t_{m-1}, \mathbf{x}\right)-c_{1}\left(t_{m-1}, \mathbf{x}\right)=e^{-r \Delta t} \iint_{\mathbb{R}^{2} \backslash\left[a_{1}, b_{1}\right] \times\left[a_{2}, b_{2}\right]} v\left(t_{m}, \mathbf{y}\right) f(\mathbf{y} \mid \mathbf{x}) d \mathbf{y}$. 
Step 2. The series truncation error:

$$
\begin{aligned}
\epsilon_{2}\left(t_{m-1}, \mathbf{x}\right) & :=c_{1}\left(t_{m-1}, \mathbf{x}\right)-c_{2}\left(t_{m-1}, \mathbf{x}\right) \\
& =\frac{b_{1}-a_{1}}{2} \frac{b_{2}-a_{2}}{2} e^{-r \Delta t} \sum_{\substack{k_{1}, k_{2} \\
k_{1} \geq N_{1} \text { or } k_{2} \geq N_{2}}}^{+\infty} A_{k_{1}, k_{2}}(\mathbf{x}) V_{k_{1}, k_{2}}\left(t_{m}\right) .
\end{aligned}
$$

The convergence rate of Fourier cosine series depends on the properties of the approximated functions in the expansion interval. The coefficients $A_{k_{1}, k_{2}}$ usually decay faster than $V_{k_{1}, k_{2}}$. With $[4,11]$, we find that the error converges exponentially in $N_{1}$ and $N_{2}$ for density functions in the class $C^{\infty}\left(\left[a_{1}, b_{1}\right] \times\left[a_{2}, b_{2}\right]\right)$. A density function with discontinuity in one of its derivatives results in an algebraic convergence of the Fourier cosine series expansion.

Step 3 . The error related to approximating $A_{k_{1}, k_{2}}(\mathbf{x})$ by $F_{k_{1}, k_{2}}(\mathbf{x})$ (see (2.6)):

$$
\begin{aligned}
\epsilon_{3}\left(t_{m-1}, \mathbf{x}\right):= & c_{2}\left(t_{m-1}, \mathbf{x}\right)-\hat{c}\left(t_{m-1}, \mathbf{x}\right) \\
= & \frac{b_{1}-a_{1}}{2} \frac{b_{2}-a_{2}}{2} e^{-r \Delta t} \sum_{k_{1}=0}^{N_{1}-1} \sum_{k_{2}=0}^{\prime N_{2}-1}\left(A_{k_{1}, k_{2}}(\mathbf{x})-F_{k_{1}, k_{2}}(\mathbf{x})\right) V_{k_{1}, k_{2}}\left(t_{m}\right) \\
= & -e^{-r \Delta t} \iint_{\mathbb{R}^{2} \backslash\left[a_{1}, b_{1}\right] \times\left[a_{2}, b_{2}\right]} \\
& {\left[\sum_{k_{1}=0}^{N_{1}-1} \sum_{k_{2}=0}^{\prime} \cos \left(k_{1} \pi \frac{y_{1}-a_{1}}{b_{1}-a_{1}}\right) \cos \left(k_{2} \pi \frac{y_{2}-a_{2}}{b_{2}-a_{2}}\right) V_{k_{1}, k_{2}}\left(t_{m}\right)\right] f(\mathbf{y} \mid \mathbf{x}) d \mathbf{y} . }
\end{aligned}
$$

Addition of the three errors gives

$$
\begin{aligned}
\epsilon\left(t_{m-1}, \mathbf{x}\right) & =e^{-r \Delta t} \iint_{\mathbb{R}^{2} \backslash\left[a_{1}, b_{1}\right] \times\left[a_{2}, b_{2}\right]}\left[v\left(t_{m}, \mathbf{y}\right)-\hat{v}\left(t_{m}, \mathbf{y}\right)\right] f(\mathbf{y} \mid \mathbf{x}) d \mathbf{y} \\
& +e^{-r \Delta t} \iint_{\left[a_{1}, b_{1}\right] \times\left[a_{2}, b_{2}\right]}\left[v\left(t_{m}, \mathbf{y}\right) f(\mathbf{y} \mid \mathbf{x})-\hat{v}\left(t_{m}, \mathbf{y}\right) \hat{f}(\mathbf{y} \mid \mathbf{x})\right] d \mathbf{y} .
\end{aligned}
$$

RESULT 1. Let us assume that the terms $V_{k_{1}, k_{2}}\left(t_{m}\right)$ are exact. If the integration domain $\left[a_{1}, b_{1}\right] \times\left[a_{2}, b_{2}\right]$ is chosen sufficiently wide, then the series truncation error $\epsilon_{2}$ dominates the overall local error. Then, for smooth density functions the error $\epsilon$ converges exponentially in $N_{1}$ and $N_{2}$; otherwise it converges algebraically. The extra error $\epsilon_{D C T}$, introduced by approximation of the terms $V_{k_{1}, k_{2}}(T)$ with DCTs, has been discussed in section 3.2.1.

We now discuss the error in the terms $V_{k_{1}, k_{2}}\left(t_{m}\right)$ and define

$$
\varepsilon_{k_{1}, k_{2}}\left(t_{m}, \mathcal{C}^{q}\right):=C_{k_{1}, k_{2}}\left(t_{m}, \mathcal{C}^{q}\right)-\hat{C}_{k_{1}, k_{2}}\left(t_{m}, \mathcal{C}^{q}\right) .
$$

The terms $G_{k_{1}, k_{2}}\left(\mathcal{G}^{p}\right)$ are assumed to be exact, so that the error in the Fourier coefficients is given by

$$
\varepsilon_{k_{1}, k_{2}}\left(t_{m}\right):=V_{k_{1}, k_{2}}\left(t_{m}\right)-\hat{V}_{k_{1}, k_{2}}\left(t_{m}\right)=\sum_{q} \varepsilon_{k_{1}, k_{2}}\left(t_{m}, \mathcal{C}^{q}\right) .
$$

RESULT 2. With $\left[a_{1}, b_{1}\right] \times\left[a_{2}, b_{2}\right] \subset \mathbb{R}^{2}$ chosen sufficiently wide and a probability density function $f$ in $C^{\infty}\left(\left[a_{1}, b_{1}\right] \times\left[a_{2}, b_{2}\right]\right)$, error $\varepsilon_{k_{1}, k_{2}}\left(t_{m}\right)$ converges exponentially

Copyright $@$ by SIAM. Unauthorized reproduction of this article is prohibited. 
in $N_{1}$ and $N_{2}$ for $1 \leq m \leq \mathcal{M}-1$. If the local error converges algebraically, then so does error $\varepsilon_{k_{1}, k_{2}}\left(t_{m}\right)$.

The proof of this result is similar to that for pricing Bermudan options with one underlying asset, which can be found in [12]. The convergence is algebraic for nonsmooth density functions or if we approximate $G_{k_{1}, k_{2}}\left(\mathcal{G}^{p}\right)$ by an algebraically converging method; see section 3.2.2.

5.2. Computational domain. The performance of the (2D-)COS method is sensitive to the choice of the computational domain $\left[a_{1}, b_{1}\right] \times\left[a_{2}, b_{2}\right]$. If the domain size is set too small, then the resulting option values may be too low. However, the larger the domain, the more terms in the series expansions that are required to reach a certain accuracy.

For the tests on rainbow options, we will take $a_{1}=a_{2}=a$ and $b_{1}=b_{2}=b$, where

$$
\begin{aligned}
& a:=\min _{i}\left[x_{0}^{i}+\xi_{1}^{i}-L \sqrt{\xi_{2}^{i}+\sqrt{\xi_{4}^{i}}}\right], \\
& b:=\max _{i}\left[x_{0}^{i}+\xi_{1}^{i}+L \sqrt{\xi_{2}^{i}+\sqrt{\xi_{4}^{i}}}\right], \quad L=10 .
\end{aligned}
$$

$\xi_{j}^{i}$ denotes the $j$ th cumulant of the stochastic variable $X_{T}^{i}$. For the cumulants $\xi_{1}, \xi_{2}$, and $\xi_{4}$ of the Brownian motion and the log-jump-diffusion process we refer the reader to [11]. The choice of equal domains for both dimensions facilitates the calculation of the coefficients $V_{k_{1}, k_{2}}(T)$.

For the Heston dynamics, we base the interval $\left[a_{1}, b_{1}\right]$ on the cumulants $\xi_{j}$ of $X_{T}$, similarly as in [11]:

$$
\left[a_{1}, b_{1}\right]:=\left[x_{0}+\xi_{1}-L \sqrt{\xi_{2}}, x_{0}+\xi_{1}+L \sqrt{\xi_{2}}\right], \quad L=8 .
$$

The second cumulant can be approximated by $\xi_{2} \approx \bar{\nu}(1+\eta) T$. For $a_{2} \geq 0$ and $b_{2}$, however, we take a tolerance level TOL $=10^{-4}$ and determine the integration range so that

$$
F_{\nu_{T} \mid \nu_{0}}\left(a_{2} \mid \nu_{0}\right)=\text { TOL }=1-F_{\nu_{T} \mid \nu_{0}}\left(b_{2} \mid \nu_{0}\right)
$$

where $F_{\nu_{T} \mid \nu_{0}}$ represents the cumulative distribution function of the variance at the terminal time. This is a general strategy in the case of nonsmooth densities.

If $N_{1}$ and $N_{2}$ are chosen sufficiently large, then a larger size of the computational domain should not affect the option price.

6. Numerical experiments for rainbow options. In this section we perform several numerical experiments to test the 2D-COS method for pricing European and Bermudan rainbow options. We will validate the algorithm and show its efficiency. The following options are studied: geometric basket, arithmetic basket, put-on-minimum, and call-on-maximum. The asset price paths are modeled by either correlated geometric Brownian motions (GBMs) or by Merton's jump-diffusion processes (but in principle we can use other Lévy processes). MATLAB 7.11.0 is used for the computations, with an Intel(R) Quad-Core $2.83 \mathrm{GHz}$ and 8 GB RAM. For the tests in this section, we take an equal number of terms in both series expansions, that is, $N_{2}=N_{1}$. 
$G B M$. Under GBM the risk-neutral asset prices evolve according to the following dynamics:

$$
d S_{t}^{i}=\left(r-\delta_{i}\right) S_{t}^{i} d t+S_{t}^{i} \sigma_{i} d Z_{t}^{i}, \quad i=1,2
$$

with correlation $d Z_{t}^{i} d Z_{t}^{j}=\rho_{i j} d t, r$ the risk-free rate, $\delta_{i}$ the dividend rate, and $\sigma_{i}$ the volatility of asset $i$. We switch to the $\log$-processes $X_{t}^{i}:=\log S_{t}^{i}$ :

$$
d X_{t}^{i}=\left(r-\delta_{i}-\frac{1}{2} \sigma_{i}^{2}\right) d t+\sigma_{i} d Z_{t}^{i}
$$

The log-asset prices at time $t_{m}$, given the values at time $t_{m-1}$, are bivariate normally distributed,

$$
\mathbf{X}_{t_{m}} \sim \mathcal{N}\left(\mathbf{X}_{t_{m-1}}+\boldsymbol{\mu}, \Sigma\right)
$$

with $\mu_{i}=\left(r-\delta_{i}-\frac{1}{2} \sigma_{i}^{2}\right) \Delta t$ and covariance matrix $\Sigma_{i j}=\sigma_{i} \sigma_{j} \rho_{i j} \Delta t$. The characteristic function reads as $\varphi(\mathbf{u} \mid \mathbf{x})=e^{i \mathbf{x}^{\prime} \mathbf{u}} \varphi_{\text {levy }}(\mathbf{u})$, with

$$
\varphi_{\text {levy }}(\mathbf{u})=\exp \left(i \boldsymbol{\mu}^{\prime} \mathbf{u}-\frac{1}{2} \mathbf{u}^{\prime} \Sigma \mathbf{u}\right)
$$

Jump-diffusion process. Under jump-diffusion the asset prices follow

$$
d S_{t}^{i}=\left(r-\lambda \varkappa_{i}\right) S_{t}^{i} d t+S_{t}^{i} \sigma_{i} d Z_{t}^{i}+\left(e^{J_{i}}-1\right) S_{t}^{i} d q_{t}, \quad i=1,2,
$$

with $\varkappa_{i}:=\mathbb{E}\left[e^{J_{i}}-1\right], q_{t}$ a Poisson process with mean arrival rate $\lambda$, and $\mathbf{J}=\left(J_{1}, J_{2}\right)$ bivariate normally distributed jumps, with mean $\boldsymbol{\mu}^{J}=\left[\mu_{1}^{J}, \mu_{2}^{J}\right]^{\prime}$ and covariance matrix $\Sigma_{i j}^{J}=\sigma_{i}^{J} \sigma_{j}^{J} \rho_{i j}^{J}$. The log-processes $X_{t}^{i}:=\log S_{t}^{i}$ read as

$$
d X_{t}^{i}=\left(r-\lambda \varkappa_{i}-\frac{1}{2} \sigma_{i}^{2}\right) d t+\sigma_{i} d Z_{t}^{i}+J_{i} d q_{t}
$$

The characteristic function reads as $\varphi(\mathbf{u} \mid \mathbf{x})=e^{i \mathbf{x}^{\prime} \mathbf{u}} \varphi_{\text {levy }}(\mathbf{u})$, with

$$
\varphi_{\text {levy }}(\mathbf{u})=\exp \left(i \boldsymbol{\mu}^{\prime} \mathbf{u}-\frac{1}{2} \mathbf{u}^{\prime} \Sigma \mathbf{u}\right) \exp \left(\lambda \Delta t\left(\exp \left(i \boldsymbol{\mu}^{\prime J} \mathbf{u}-\frac{1}{2} \mathbf{u}^{\prime} \Sigma^{J} \mathbf{u}\right)-1\right)\right)
$$

where $\mu_{i}=\left(r-\lambda \varkappa_{i}-\frac{1}{2} \sigma_{i}^{2}\right) \Delta t$ and $\Sigma_{i j}=\sigma_{i} \sigma_{j} \rho_{i j} \Delta t$.

The bivariate density functions of the correlated Brownian motion and jumpdiffusion process are both in $C^{\infty}$, which will result in exponentially converging errors in $N_{1}$ and $N_{2}$. Density recovery using Fourier cosine expansions and the characteristic function gives

$$
\hat{f}(\mathbf{y} \mid \mathbf{x}):=\sum_{k_{1}=0}^{N_{1}-1} \sum_{k_{2}=0}^{\prime} F_{2}-1
$$

The approximated density function of a log-jump-diffusion process is presented in Figure 6.1. 

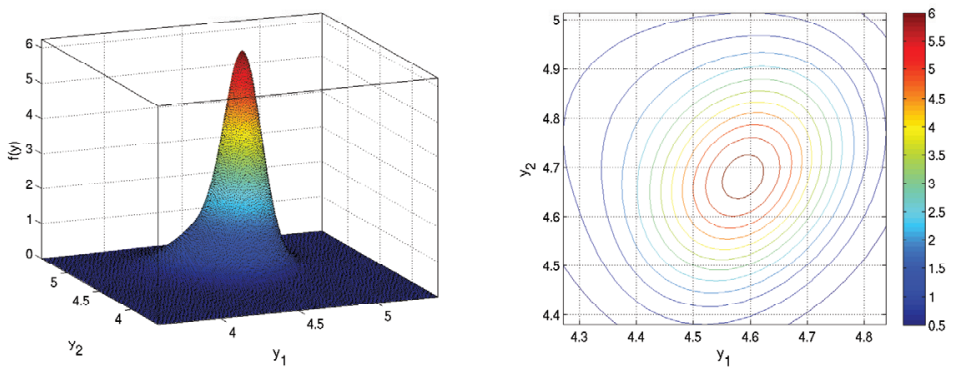

FIG. 6.1. Density recovery jump-diffusion process $\hat{f}\left(\mathbf{X}_{T} \mid \mathbf{x}_{0}\right)$ (parameters from Set II).

Parameter sets. The following five parameters sets are used for the rainbow options.

$\underline{\text { Set I: }}$

$\mathbf{S}_{0}=\left[\begin{array}{ll}90 & 110\end{array}\right]^{\prime}, r=0.04, \delta_{i}=0, \sigma=\left[\begin{array}{cc}0.2 & 0.3\end{array}\right], \rho=\left[\begin{array}{cc}1 & 0.25 \\ 0.25 & 1\end{array}\right], t_{0}=0, T=1, K=100$. $\underline{\text { Set II: }}$

$\mathbf{S}_{0}=\left[\begin{array}{ll}90 & 110\end{array}\right]^{\prime}, r=0.05, \sigma=\left[\begin{array}{ll}0.12 & 0.15\end{array}\right], \rho=\left[\begin{array}{cc}1 & 0.30 \\ 0.30 & 1\end{array}\right], t_{0}=0, T=1, K=100$, $\lambda=0.60, \boldsymbol{\mu}^{J}=[-0.10,0.10]^{\prime}, \sigma^{J}=\left[\begin{array}{ll}0.17 & 0.13\end{array}\right], \rho^{J}=\left[\begin{array}{cc}1 & -0.20 \\ -0.20 & 1\end{array}\right]$.

Set III:

$\mathbf{S}_{0}=\left[\begin{array}{ll}40 & 40\end{array}\right]^{\prime}, r=0.048790, \delta_{i}=0, \sigma=[0.2 \quad 0.3], \rho=\left[\begin{array}{cc}1 & 0.5 \\ 0.5 & 1\end{array}\right], t_{0}=0, T=7 / 12$, $K=40$.

Set IV:

$\mathbf{S}_{0}=\left[\begin{array}{lll}100 & 100 & 100\end{array}\right]^{\prime}, r=0.04, \delta_{i}=0, \sigma=\left[\begin{array}{lll}0.3 & 0.35 & 0.4\end{array}\right], \rho=\left[\begin{array}{ccc}1 & 0.5 & 0.5 \\ 0.5 & 1 & 0.5 \\ 0.5 & 0.5 & 1\end{array}\right], t_{0}=0$, $T=1, K=100$.

$\underline{\text { Set V: }}$

$$
r=0.05, \delta_{i}=0.1, \sigma=\left[\begin{array}{ll}
0.2 & 0.2
\end{array}\right], \rho=\left[\begin{array}{ll}
1 & 0 \\
0 & 1
\end{array}\right], t_{0}=0, T=3, K=100 .
$$

6.1. European rainbow options. We start in sections 6.1 .1 and 6.1 .2 by pricing two types of basket options with two underlying asset prices, namely,

- geometric basket call options, with payoff $g(\mathbf{x})=\left(\sqrt{e^{x_{1}}} \sqrt{e^{x_{2}}}-K\right)^{+}$,

- arithmetic basket call options, with payoff $g(\mathbf{x})=\left(\frac{1}{2} e^{x_{1}}+\frac{1}{2} e^{x_{2}}-K\right)^{+}$. Then, section 6.1.3 discusses call-on-maximum and put-on-minimum options.

6.1.1. Geometric basket call option. The price of a geometric basket call option under GBM equals the price of a Black-Scholes call option with initial option price $\hat{S}_{0}=\sqrt{S_{0}^{1}} \sqrt{S_{0}^{2}}$, dividend rate $\hat{\delta}$, and volatility $\hat{\sigma}$ [22], where

$$
\hat{\sigma}=\sqrt{\left(\frac{1}{2}\right)^{2} \sum_{i, j} \sigma_{i} \sigma_{j} \rho_{i j}} \quad \text { and } \quad \hat{\delta}=\frac{1}{2} \sum_{i}\left(\delta_{i}+\frac{1}{2} \sigma_{i}^{2}\right)-\frac{1}{2} \hat{\sigma}^{2} .
$$

Copyright $@$ by SIAM. Unauthorized reproduction of this article is prohibited. 
So, we can compare our results with the analytic option values. The Fourier cosine coefficients of the payoff function are given by

$$
\begin{aligned}
V_{k_{1}, k_{2}}(T)= & \frac{2}{b_{1}-a_{1}} \frac{2}{b_{2}-a_{2}} \int_{a_{2}}^{b_{2}} \int_{a_{1}}^{b_{1}}\left(\sqrt{e^{y_{1}}} \sqrt{e^{y_{2}}}-K\right)^{+} \\
& \cos \left(k_{1} \pi \frac{y_{1}-a_{1}}{b_{1}-a_{1}}\right) \cos \left(k_{2} \pi \frac{y_{2}-a_{2}}{b_{2}-a_{2}}\right) d y_{1} d y_{2} .
\end{aligned}
$$

An analytic solution is available and can be found using, for instance, Maple 14. We use parameter set I. Besides, we test the method for a deep out-of-the-money option with strike price $K=200$. The option values are $v\left(t_{0}, \mathbf{x}_{0}\right)=8.8808(K=100)$ and $v\left(t_{0}, \mathbf{x}_{0}\right)=2.8 \times 10^{-3}(K=200)$. The results in Table 2 and Figure 6.2 are highly satisfactory and show exponential convergence. Convergence is reached in milliseconds. For both strike prices, the Fourier coefficients $V_{k_{1}, k_{2}}$ are of the same order of magnitude. The last coefficients retained in the series expansion truncation determine the order of magnitude of the truncation error. Because of that, the error results are similar; however, the relative error is larger for the out-of-the-money option.

TABLE 2

Results for the geometric basket call (GBM).

\begin{tabular}{l|cccc}
\hline$N_{1}\left(=N_{2}\right)$ & 10 & 20 & 40 & 80 \\
\hline Error $(K=100)$ & $-7.60 \mathrm{e}-1$ & $-4.07 \mathrm{e}-2$ & $-1.42 \mathrm{e}-5$ & $2.34 \mathrm{e}-13$ \\
Error $(K=200)$ & $-7.23 \mathrm{e}-1$ & $-4.06 \mathrm{e}-2$ & $-1.42 \mathrm{e}-5$ & $3.08 \mathrm{e}-13$ \\
CPU $(\mathrm{ms})$ & 1.65 & 1.99 & 3.15 & 7.46 \\
\hline
\end{tabular}

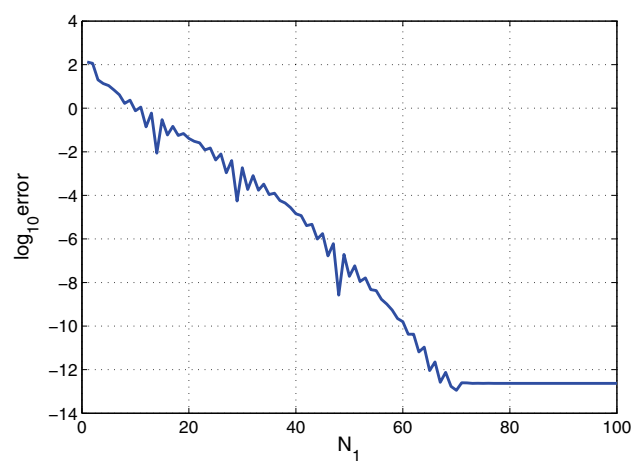

FIG. 6.2. Error for the geometric basket call $(K=100)(G B M)$.

In section 6.1.2, we will price arithmetic basket options, for which the terminal coefficients $V_{k_{1}, k_{2}}(T)$ need to be approximated by means of 2D discrete Fourier cosine transforms, as explained in section 3.2.1. Since there is an analytic solution available for the geometric basket options, we can analyze the error of the discretization approach for this option. For each spatial dimension we now take $Q \geq N_{1}$ grid-points. So we replace the payoff coefficients by a discrete approximation.

First, we analyze the approximate coefficients $V_{k_{1}, k_{2}}^{D C T}(T)$ for the geometric basket option and calculate the maximum absolute error. This error converges quadratically in $Q$; see Figure 6.3. Second, we calculate the errors of the COS formula; see section 5.1. The computational domain is chosen sufficiently large, so that we can neglect 


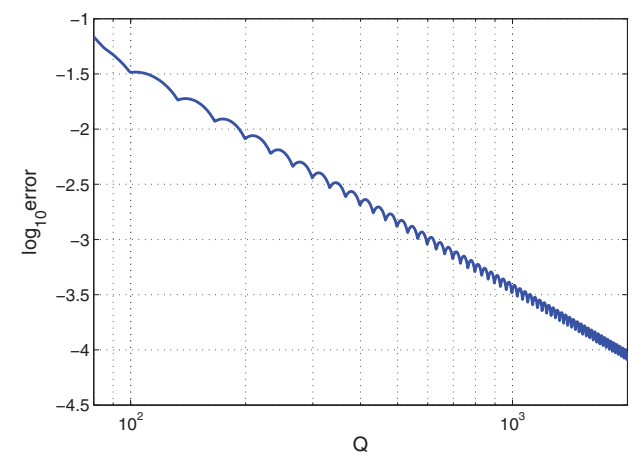

FIG. 6.3. Maximum absolute error $V_{k_{1}, k_{2}}^{D C T}(T)\left(k_{1}, k_{2}=1, \ldots, 80\right)$.

TABLE 3

Results for the geometric basket call option, with payoff coefficients approximated by DCTs.

(a) Absolute error.

\begin{tabular}{rr|cccc}
\hline & & \multicolumn{4}{|c}{$N_{1}\left(=N_{2}\right)$} \\
& & 10 & 20 & 40 & 80 \\
\hline \multirow{4}{*}{$Q$} & 250 & $1.09 \mathrm{e}+1$ & $7.61 \mathrm{e}-1$ & $4.18 \mathrm{e}-2$ & $1.14 \mathrm{e}-3$ \\
& 500 & $7.59 \mathrm{e}-1$ & $4.02 \mathrm{e}-2$ & $3.91 \mathrm{e}-4$ & $4.05 \mathrm{e}-4$ \\
& 1000 & $7.60 \mathrm{e}-1$ & $4.06 \mathrm{e}-2$ & $4.96 \mathrm{e}-5$ & $6.38 \mathrm{e}-5$ \\
& 2000 & $7.60 \mathrm{e}-1$ & $4.07 \mathrm{e}-2$ & $1.36 \mathrm{e}-5$ & $5.75 \mathrm{e}-7$ \\
\hline
\end{tabular}

(b) CPU time (in seconds).

\begin{tabular}{rr|cccc}
\hline & & \multicolumn{4}{|c}{$N_{1}\left(=N_{2}\right)$} \\
& & 10 & 20 & 40 & 80 \\
\hline \multirow{4}{*}{$Q$} & 250 & 0.007 & 0.007 & 0.008 & 0.014 \\
& 500 & 0.04 & 0.04 & 0.04 & 0.06 \\
& 1000 & 0.17 & 0.17 & 0.17 & 0.20 \\
& 2000 & 0.71 & 0.71 & 0.73 & 0.75 \\
\hline
\end{tabular}
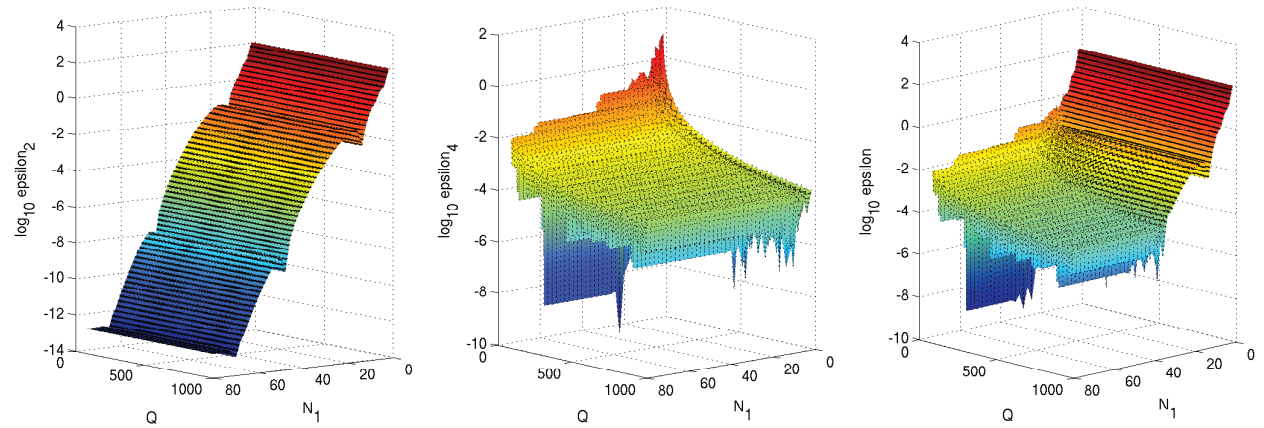

FIG. 6.4. Error for the geometric basket call option, with payoff coefficients approximated by DCTs.

errors $\epsilon_{1}$ and $\epsilon_{3}$, in (5.2) and (5.4), respectively. Results are shown in Table 3 and Figure 6.4. The left plot shows the series truncation error $\epsilon_{2}$, which indeed converges exponentially in $N_{1}$. The midplot confirms the algebraically converging error $\epsilon_{D C T}$, and the right plot shows the total error $\epsilon$. If $N_{1}$ is chosen sufficiently large, then the error of using discrete Fourier transforms dominates the total error. The computation time of the coefficients is of order $O\left(Q^{2} \log _{2} Q\right)$ and is the most time-consuming part. The CPU time is, however, still less than one second.

6.1.2. Arithmetic basket call option. For arithmetic basket options under GBM, there is no analytic solution to the option price. Instead, we use the reference option value $v\left(t_{0}, \mathbf{x}_{0}\right) \approx 10.173230$, obtained by using $Q=5000, N_{1}=N_{2}=100$ 
(Set I). This value is validated by a plain Monte Carlo simulation with $10^{6}$ runs, which results in $v\left(t_{0}, \mathbf{x}_{0}\right) \approx 10.1714$ with standard deviation 0.017 .

The Fourier cosine coefficients of the payoff function are given by

$$
\begin{aligned}
V_{k_{1}, k_{2}}(T)= & \frac{2}{b_{1}-a_{1}} \frac{2}{b_{2}-a_{2}} \int_{a_{2}}^{b_{2}} \int_{a_{1}}^{b_{1}}\left(\frac{1}{2} e^{y_{1}}+\frac{1}{2} e^{y_{2}}-K\right)^{+} \\
& \cos \left(k_{1} \pi \frac{y_{1}-a_{1}}{b_{1}-a_{1}}\right) \cos \left(k_{2} \pi \frac{y_{2}-a_{2}}{b_{2}-a_{2}}\right) d y_{1} d y_{2} .
\end{aligned}
$$

No analytic representation is available, and we approximate the coefficients by using DCTs. The results in Table 4 are satisfactory, and the error converges exponentially in $N_{1}$. The CPU times are the same as in Table 3 , and convergence is achieved within 2 seconds.

TABLE 4

Absolute error for the arithmetic basket call option.

\begin{tabular}{rr|cccc}
\hline & & \multicolumn{4}{c}{$N_{1}\left(=N_{2}\right)$} \\
& & 10 & 20 & 40 & 80 \\
\hline \multirow{4}{*}{$Q$} & 250 & $2.97 \mathrm{e} 0$ & $1.85 \mathrm{e}-1$ & $2.65 \mathrm{e}-3$ & $2.70 \mathrm{e}-3$ \\
& 500 & $2.98 \mathrm{e} 0$ & $1.91 \mathrm{e}-1$ & $1.97 \mathrm{e}-5$ & $2.97 \mathrm{e}-5$ \\
& 1000 & $2.98 \mathrm{e} 0$ & $1.91 \mathrm{e}-1$ & $4.82 \mathrm{e}-5$ & $1.29 \mathrm{e}-6$ \\
& 2000 & $2.98 \mathrm{e} 0$ & $1.91 \mathrm{e}-1$ & $5.08 \mathrm{e}-5$ & $1.28 \mathrm{e}-6$ \\
\hline
\end{tabular}

6.1.3. Call-on-max and put-on-min options. In this section, we discuss the following two-color rainbow options:

- call-on-maximum option, with payoff $g(\mathbf{x})=\left(\max \left(e^{x_{1}}, e^{x_{2}}\right)-K\right)^{+}$,

- put-on-minimum option, with payoff $g(\mathbf{x})=\left(K-\min \left(e^{x_{1}}, e^{x_{2}}\right)\right)^{+}$.

The Fourier coefficients of the payoff functions can be calculated analytically. Next, we summarize the results. For the European call-on-max and put-on-min options under GBM we use the parameters from Set III [5] and compare the results with the analytic solutions from [27]. Figure 6.5 shows the results and again confirms exponential convergence.
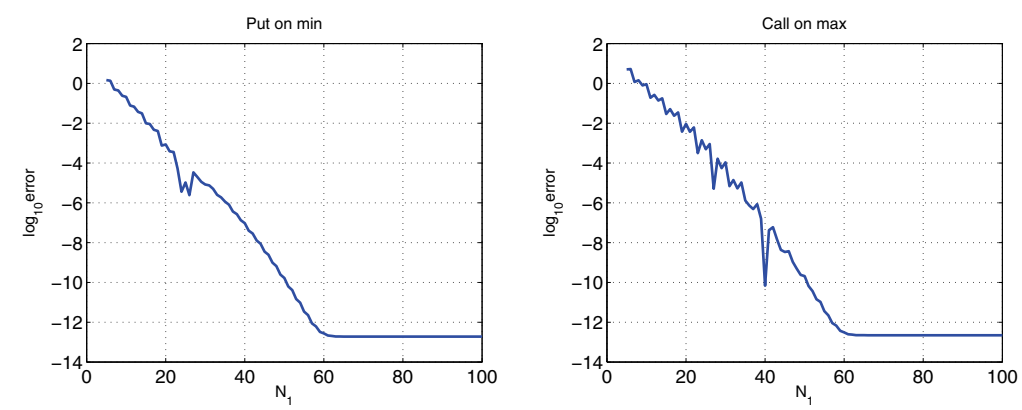

FIG. 6.5. Error for put-on-min and call-on-max options.

Next we consider a put-on-minimum option under a jump-diffusion process. Under jump-diffusion asset price processes, there is no analytic solution to the option values available. We use the model parameters from Set II and set $N_{1}=N_{2}=125$, for which machine precision is reached. The results in Table 5 are achieved in a few milliseconds and correspond well to the reference prices in [8]. 
TABLE 5

Put-on-min option values $\hat{v}\left(t_{0}, \mathbf{x}_{0}\right)$ (jump-diffusion).

\begin{tabular}{cc|ccc}
\hline$S_{0}^{2}$ & $S_{0}^{1}$ & 90 & 100 & 110 \\
\hline 90 & 15.6916 & 13.4073 & 12.1305 \\
100 & 12.1918 & 9.1360 & 7.5175 \\
110 & 10.3853 & 6.7274 & 4.8337 \\
\hline
\end{tabular}

6.2. Bermudan rainbow options. For pricing Bermudan options we need to determine rectangular continuation and early-exercise regions. For this, we divide the domain of the second dimension, $\left[a_{2}, b_{2}\right]$, into $J$ subintervals:

$$
\left[a_{2}, b_{2}\right]=\left[w_{0}, w_{1}\right] \cup\left[w_{1}, w_{2}\right] \ldots\left[w_{q}, w_{q+1}\right] \ldots\left[w_{J-1}, w_{J}\right] .
$$

At the center of each subinterval, we determine the value(s) $y^{*}$ for which the optimal exercise policy changes to the optimal continuation, i.e.,

$$
g\left(y^{*}, \frac{1}{2}\left(w_{q}+w_{q+1}\right)\right)=c\left(t_{m}, y^{*}, \frac{1}{2}\left(w_{q}+w_{q+1}\right)\right) .
$$

For, for instance, a basket put option, we can then define an early-exercise region $\mathcal{G}^{q}=\left[a_{1}, y^{*}\right] \times\left[w_{q}, w_{q+1}\right]$ and a continuation region $\mathcal{C}^{q}=\left[y^{*}, b_{1}\right] \times\left[w_{q}, w_{q+1}\right]$. Therefore, the computational domain is divided into $J$ early-exercise and continuation regions. In the case of a call-on-max option there is a multiply connected exercise region (see Figure 3.1 ), and for each subinterval $\left[w_{q}, w_{q+1}\right]$ we have one or two earlyexercise regions $\mathcal{G}^{q}$. As long as we select a technique by which we can distinguish multiple continuation and early-exercise regions from each other, this does not affect the complexity of the method.

Remark 4. We elaborate on the error in the determination of the early-exercise regions and the convergence in parameter $J$. We assume that $N_{1}$ and $N_{2}$ are chosen sufficiently large, so that the approximated option values are sufficiently accurate. Besides, we presume that a root-finding method is available which is able to accurately determine the value(s) $y^{*}$; see (6.12). For example, the Newton method with an accurate initial guess suffices. In this setting, the early-exercise regions converge, quadratically in $J$, to the true regions by means of the rectangle-rule-based method. However, if we would choose $N_{1}, N_{2}$ too small, the COS formula might give inaccurate, oscillatory options values, resulting in inaccurate early-exercise regions and the convergence result holding only for sufficiently large $N_{1}, N_{2}$.

For equidistant intervals $\left[w_{q}, w_{q+1}\right]$ we take $w_{q}=a_{2}+\left(b_{2}-a_{2}\right) q / J$, which gives the exercise regions $\mathcal{G}^{q}$ shown in Figure 6.6(a). A nonequidistant grid, as in Figure 6.6(b), is based on the quantile function (the inverse distribution function) of asset price $X_{T}^{2}$. We use $w_{0}=a_{2}, w_{J}=b_{2}$, and $w_{q}=F_{X_{T}^{2}}^{-1}(q / J)$. The large rectangle at the bottom of the domain is not an accurate representation of the true region. However, it is located near the boundary of the computational domain, outside the domain of interest, and therefore does not affect the option values in the middle of the domain. The usage of nonequidistant grids typically leads to more efficient pricing.

Remark 5. If the quantile function is not known analytically, then we can approximate the random variable by a normally distributed variable with the same mean (first cumulant) and variance (second cumulant), for which the quantile function is known.

6.2.1. Geometric basket put option. We start by pricing Bermudan-style geometric basket put options under GBM. For the tests we use the parameters from 


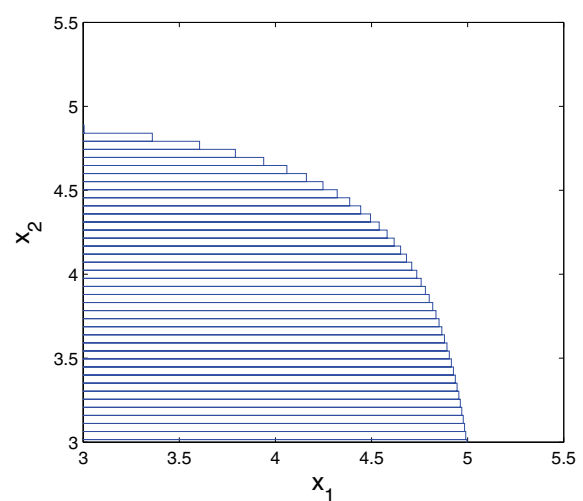

(a) Equidistant grid.

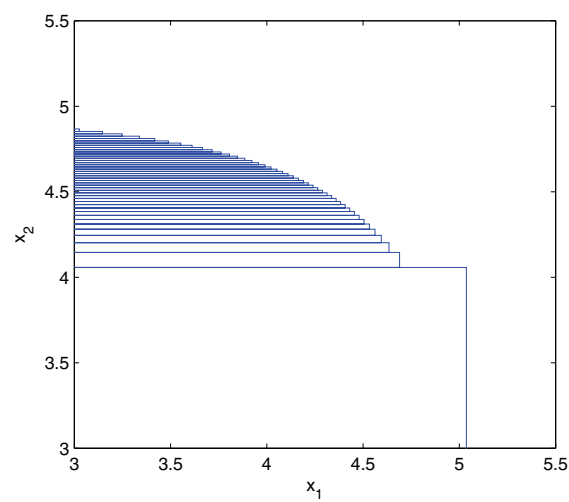

(b) Nonequidistant grid.

FIG. 6.6. Rectangular early-exercise regions $\mathcal{G}^{q}$ (arithmetic basket put).

Set I and take $\mathcal{M}=10$ early-exercise dates. The reference solution to the put option, $v\left(t_{0}, \mathbf{x}\right)=6.95904$, equals a Bermudan put option in one dimension [3] and is computed with the $1 \mathrm{D}$-COS method. The terms $G_{k_{1}, k_{2}}\left(\mathcal{G}^{p}\right)$ admit an analytic solution. The results are presented in Table 6 . Convergence is exponential in $N_{1}$ and algebraic in $J$, with order two; see section 5.1. The use of nonequidistant grids improves the convergence significantly. The computation time is linear in $J$, and the results for, for example, $N_{1}=80$ and $J=160$ are obtained in about 30 seconds.

TABLE 6

Absolute error for the Bermudan geometric put basket option.

(a) Equidistant grid.

\begin{tabular}{rr|ccc}
\hline & & \multicolumn{3}{|c}{$N_{1}\left(=N_{2}\right)$} \\
& & 40 & 80 & 160 \\
\hline \multirow{4}{*}{$J$} & 20 & $6.65 \mathrm{e}-2$ & $7.77 \mathrm{e}-2$ & $7.79 \mathrm{e}-2$ \\
& 40 & $1.22 \mathrm{e}-2$ & $1.56 \mathrm{e}-2$ & $1.56 \mathrm{e}-2$ \\
& 80 & $1.69 \mathrm{e}-4$ & $3.54 \mathrm{e}-3$ & $3.51 \mathrm{e}-3$ \\
& 160 & $2.51 \mathrm{e}-3$ & $8.86 \mathrm{e}-4$ & $8.56 \mathrm{e}-4$ \\
& 320 & $3.15 \mathrm{e}-3$ & $2.42 \mathrm{e}-4$ & $2.13 \mathrm{e}-4$ \\
\hline
\end{tabular}

(b) Nonequidistant grid.

\begin{tabular}{rr|ccc}
\hline & & \multicolumn{3}{|c}{$N_{1}\left(=N_{2}\right)$} \\
& & 40 & 80 & 160 \\
\hline \multirow{4}{*}{$J$} & 20 & $7.07 \mathrm{e}-4$ & $2.23 \mathrm{e}-3$ & $2.20 \mathrm{e}-3$ \\
& 40 & $2.63 \mathrm{e}-3$ & $5.13 \mathrm{e}-4$ & $4.83 \mathrm{e}-4$ \\
& 80 & $2.97 \mathrm{e}-3$ & $1.46 \mathrm{e}-4$ & $1.16 \mathrm{e}-4$ \\
& 160 & $3.17 \mathrm{e}-3$ & $5.85 \mathrm{e}-5$ & $2.88 \mathrm{e}-5$ \\
& 320 & $3.18 \mathrm{e}-3$ & $3.70 \mathrm{e}-5$ & $7.17 \mathrm{e}-6$ \\
\hline
\end{tabular}

In section 6.2.2, we will price Bermudan arithmetic basket options, for which the coefficients $V_{k_{1}, k_{2}}(T)$ are approximated by means of 2D DCTs and the terms $G_{k_{1}, k_{2}}$ are approximated by $\hat{G}_{k_{1}, k_{2}}$, as explained in section 3.2 .2 (by (3.25)). We examine here the error of this approach by using the Bermudan geometric basket, for which an accurate reference price is available. We take $Q=2000$. Table 7 shows satisfactorily results. The results are similar to those of Table 6 , with analytic terms $G_{k_{1}, k_{2}}$. However, for higher values of $N_{1}$ the usage of analytic terms is more accurate.

6.2.2. Arithmetic basket put option. For the arithmetic basket option, we take the same model parameters (Set I) as in the previous section, with $\mathcal{M}=10$ early-exercise dates. We approximate the Fourier coefficients at the terminal time by using the DCT, and the coefficients $G_{k_{1}, k_{2}}$ are approximated by $\hat{G}_{k_{1}, k_{2}}$. The results in Table 8 show converging option values. 
TABLE 7

Absolute error for the Bermudan geometric put basket option, with $\hat{G}_{k_{1}, k_{2}}(Q=2000)$.

(a) Equidistant grid.

\begin{tabular}{rr|ccc}
\hline & & \multicolumn{3}{|c}{$N_{1}\left(=N_{2}\right)$} \\
& & 40 & 80 & 160 \\
\hline \multirow{4}{*}{$J$} & 20 & $7.05 \mathrm{e}-02$ & $7.78 \mathrm{e}-02$ & $7.79 \mathrm{e}-02$ \\
& 40 & $1.70 \mathrm{e}-02$ & $1.58 \mathrm{e}-02$ & $1.56 \mathrm{e}-02$ \\
& 80 & $5.56 \mathrm{e}-03$ & $3.69 \mathrm{e}-03$ & $3.54 \mathrm{e}-03$ \\
& 160 & $2.96 \mathrm{e}-03$ & $1.09 \mathrm{e}-03$ & $9.13 \mathrm{e}-04$ \\
& 320 & $2.33 \mathrm{e}-03$ & $4.66 \mathrm{e}-04$ & $2.86 \mathrm{e}-04$ \\
\hline
\end{tabular}

(b) Nonequidistant grid.

\begin{tabular}{rr|ccc}
\hline & & \multicolumn{3}{|c}{$N_{1}\left(=N_{2}\right)$} \\
& & 40 & 80 & 160 \\
\hline \multirow{4}{*}{$J$} & 20 & $4.55 \mathrm{e}-03$ & $2.45 \mathrm{e}-03$ & $2.25 \mathrm{e}-03$ \\
& 40 & $2.69 \mathrm{e}-03$ & $7.39 \mathrm{e}-04$ & $5.54 \mathrm{e}-04$ \\
& 80 & $2.37 \mathrm{e}-03$ & $3.74 \mathrm{e}-04$ & $1.92 \mathrm{e}-04$ \\
& 160 & $2.23 \mathrm{e}-03$ & $2.87 \mathrm{e}-04$ & $1.06 \mathrm{e}-04$ \\
& 320 & $2.21 \mathrm{e}-03$ & $2.66 \mathrm{e}-04$ & $8.52 \mathrm{e}-05$ \\
\hline
\end{tabular}

TABLE 8

Option values for the Bermudan arithmetic put basket option, with $\hat{G}_{k_{1}, k_{2}}(Q=2000)$.

\begin{tabular}{rr|ccc}
\hline \multicolumn{4}{c}{ Nonequidistant grid } \\
\multicolumn{4}{c|}{} & \multicolumn{3}{c}{$N_{1}\left(=N_{2}\right)$} \\
\hline \multirow{4}{*}{$J$} & 20 & 6.6086 & 6.6067 & 6.6077 \\
& 40 & 6.6102 & 6.6091 & 6.6102 \\
& 80 & 6.6106 & 6.6096 & 6.6108 \\
& 160 & 6.6109 & 6.6097 & 6.6109 \\
& 320 & 6.6108 & 6.6097 & 6.6109 \\
\hline
\end{tabular}

6.2.3. Call-on-max and put-on-min options. Here, we consider Bermudan call-on-maximum and put-on-minimum options. They are discussed in, among others, $[2,8,16,15]$. The authors in $[7]$ prove properties of the continuation and early-exercise regions for the American-style option.

The convergence results for a call-on-max option under GBM, Set V with $\mathcal{M}=9$, are presented in Table 9(a). In Table 9(b), option prices for different initial asset values are shown. They correspond to the values in the third and fourth columns, which are obtained in [2].

TABLE 9

Bermudan call-on-max option values $\hat{v}\left(t_{0}, \mathbf{x}\right)$.

(b) Option prices for different initial values $\left(S_{0}^{2}=S_{0}^{1}\right)$

(a) Convergence in $N_{1}$ and $J$. $\left(N_{1}=160, J=320\right)$.

\begin{tabular}{|c|c|c|c|c|c|c|c|c|}
\hline & \multicolumn{3}{|c|}{$N_{1}\left(=N_{2}\right)$} & \multirow[b]{2}{*}{$S_{0}^{1}$} & \multirow[b]{2}{*}{ 2D-COS } & \multirow[b]{2}{*}{$95 \%$ CI [2] } & \multirow[b]{2}{*}{ Binomial [2] } \\
\hline & & 40 & 80 & 160 & & & & \\
\hline \multirow{5}{*}{$J$} & 40 & 14.2946 & 13.8919 & 13.8921 & 90 & 8.0727 & {$[8.053,8.082]$} & 8.075 \\
\hline & 80 & 14.3032 & 13.8993 & 13.8994 & 100 & 13.9017 & {$[13.892,13.934]$} & 13.902 \\
\hline & 160 & 14.3025 & 13.9011 & 13.9013 & 110 & 21.3437 & {$[21.316,21.359]$} & 21.345 \\
\hline & 320 & 14.3029 & 13.9015 & 13.9017 & & & & \\
\hline & 640 & 14.3037 & 13.9016 & 13.9018 & & & & \\
\hline
\end{tabular}

At last, we price put-on-minimum options under a jump-diffusion process (parameters from Set II). The results in Table 10(a) show converging values. This verifies the applicability of the 2D-COS method to the broad class of Lévy processes. With increasing the number of early-exercise dates, $\mathcal{M}$, the option prices converge to the American prices in [8] (Table 10(b)).

Remark 6 . Other rainbow options that can by priced easily and efficiently with the 2D-COS formula are, among others, double binary (digital) cash-or-nothing options, spread options, two-asset correlation options, and exchange options.

Copyright $@$ by SIAM. Unauthorized reproduction of this article is prohibited. 
TABLE 10

Bermudan put-on-min option values $\hat{v}\left(t_{0}, \mathbf{x}\right)$ (jump-diffusion).

(b) Convergence in $\mathcal{M}$

(a) Convergence in $N_{1}$ and $J(\mathcal{M}=9)$.

$\left(J=320, N_{1}=160\right)$.

\begin{tabular}{rr|ccc}
\hline & & \multicolumn{3}{|c}{$N_{1}\left(=N_{2}\right)$} \\
& & 40 & 80 & 160 \\
\hline \multirow{4}{*}{$J$} & 40 & 9.5495 & 9.5570 & 9.5586 \\
& 80 & 9.5597 & 9.5580 & 9.5597 \\
& 160 & 9.5606 & 9.5582 & 9.5599 \\
& 320 & 9.5621 & 9.5584 & 9.5600 \\
& 640 & 9.5632 & 9.5584 & 9.5600 \\
\hline
\end{tabular}

\begin{tabular}{r|l}
$\mathcal{M}$ & \\
\hline 2 & 9.3577 \\
4 & 9.4863 \\
8 & 9.5526 \\
16 & 9.5862 \\
32 & 9.6033 \\
\hline
\end{tabular}

7. Numerical experiments under the Heston model. In this section, we test the performance of the 2D-COS method for pricing Bermudan put options under the Heston dynamics. We use three different parameter sets:

Set A: $\rho=0.1, \nu_{0}=0.0625, \bar{\nu}=0.16, S_{0}=10, K=10, r=0.1, \eta=0.9, \kappa=5, T=0.25$.

Set B: $\rho=-0.64, \nu_{0}=0.0348, \bar{\nu}=0.0348, S_{0}=100, K=100, r=0.04$,

$$
\eta=0.9, \kappa=1.15, T=0.25 \text {. }
$$

Set C: $\rho=-0.9, \nu_{0}=0.04, \bar{\nu}=0.04, S_{0}=100, K=100, r=0.04, \eta=0.5, \kappa=0.5, T=1$.

The Feller condition satisfied is equivalent to $2 \kappa \bar{\nu} / \eta^{2}-1:=q_{F} \geq 0$. For Set A we have $q_{F}=0.98$ and the variance process remains strictly positive. For sets $\mathrm{B}$ and $\mathrm{C}$ we have $q_{F}=-0.47$ and $q_{F}=-0.84$, respectively, and the Feller condition is not satisfied.

Figure 7.1 shows the approximated bivariate density function $\hat{f}\left(X_{T}, \nu_{T} \mid x_{0}, \nu_{0}\right)$, as obtained by density recovery, (6.8), for Set A.
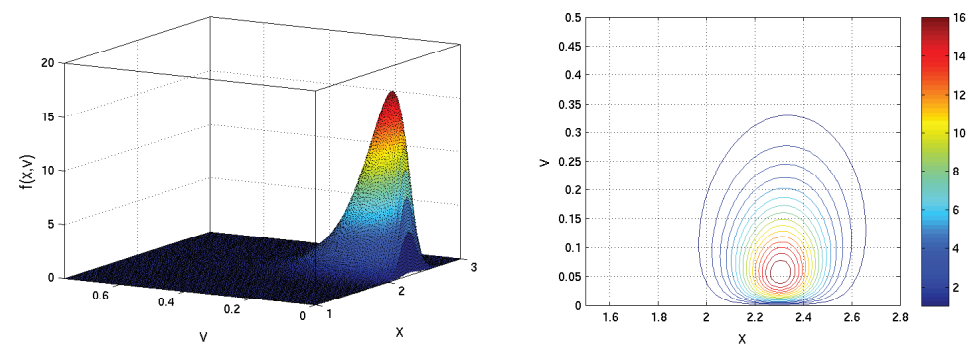

FIG. 7.1. Bivariate density function (Set A).

We find that

$$
\left|F_{k_{1}, k_{2}}^{ \pm}(\mathbf{x})\right| \leq \frac{2}{b_{1}-a_{1}} \frac{2}{b_{2}-a_{2}}\left|\varphi\left(\frac{k_{1} \pi}{b_{1}-a_{1}}, \pm \frac{k_{2} \pi}{b_{2}-a_{2}} \mid \mathbf{x}\right)\right| \sim O\left(k_{2}^{-\left(q_{F}+1\right)}\right)
$$

based on the univariate characteristic function of the variance process. The coefficients $F_{k_{1}, k_{2}}$ decrease exponentially in $k_{1}$ and algebraically in $k_{2}$ with order $q_{F}+1$. For Set $\mathrm{C}$ this yields convergence order 0.16 , which will result in very slow convergence of the 2D-COS method in this case.

7.1. European options with Bermudan framework. To test the algorithm for pricing under the Heston stochastic volatility model, we calculate European option prices with the Bermudan framework, with $\mathcal{M}=12$ time steps. At every time step 
$t_{m}$ we take only a continuation region, $\mathcal{C}^{1}=\left[a_{1}, b_{1}\right] \times\left[a_{2}, b_{2}\right]$, which then corresponds to a European option and no early-exercise opportunities.

Reference prices are obtained by the 1D-COS method for European options [11]. The error results are shown in Table 11. The error converges exponentially in $N_{1}$ and algebraically in $N_{2}$, as expected. Sets A and B give very accurate prices. However, the convergence for Set $\mathrm{C}$ is relatively slow, and the obtained values are not highly accurate. The reason for this is the peaked density function, with coefficients $F_{k_{1}, k_{2}}$ only slowly decreasing in $k_{2}$.

TABLE 11

Error for European option prices.

(b) Set $\mathrm{B}, v\left(t_{0}, \mathbf{x}\right)=3.1325\left(q_{F}=\right.$

(a) Set A, $\left.v\left(t_{0}, \mathbf{x}\right)=0.5015\left(q_{F}=0.98\right) . \quad-0.47\right)$.

\begin{tabular}{|c|c|c|c|c|c|c|c|}
\hline \multirow[t]{2}{*}{$N_{1}$} & \multicolumn{3}{|c|}{$\overline{N_{2}}$} & \multirow[t]{2}{*}{$N_{1}$} & \multicolumn{3}{|c|}{$\mathrm{N}_{2}$} \\
\hline & 50 & 100 & 200 & & 50 & 100 & 200 \\
\hline 50 & $-1.01 \mathrm{e}-4$ & $-1.03 \mathrm{e}-5$ & $1.02 \mathrm{e}-6$ & 50 & $3.83 \mathrm{e}-4$ & $2.05 \mathrm{e}-4$ & $8.94 \mathrm{e}-5$ \\
\hline 100 & $-1.07 \mathrm{e}-4$ & $-1.63 \mathrm{e}-5$ & $-4.90 \mathrm{e}-6$ & 100 & $3.75 e-4$ & $1.95 \mathrm{e}-4$ & $7.90 \mathrm{e}-5$ \\
\hline 200 & $-1.07 \mathrm{e}-4$ & $-1.62 \mathrm{e}-5$ & $-4.86 \mathrm{e}-6$ & 200 & $3.75 e-4$ & $1.95 \mathrm{e}-4$ & $7.90 \mathrm{e}-5$ \\
\hline
\end{tabular}

(c) Set C, $v\left(t_{0}, \mathbf{x}\right)=6.2711\left(q_{F}=-0.84\right)$.

\begin{tabular}{r|ccc}
\hline$N_{1}$ & & $N_{2}$ & \\
& 250 & 500 & 1000 \\
\hline 50 & $7.8787 \mathrm{e}-02$ & $4.3008 \mathrm{e}-02$ & $2.6131 \mathrm{e}-02$ \\
100 & $6.8267 \mathrm{e}-02$ & $3.1137 \mathrm{e}-02$ & $1.3630 \mathrm{e}-02$ \\
200 & $6.7979 \mathrm{e}-02$ & $3.0760 \mathrm{e}-02$ & $1.3220 \mathrm{e}-02$ \\
\hline
\end{tabular}

7.2. Bermudan put options under Heston dynamics. In this section, we consider Bermudan put options with $\mathcal{M}=10$ early-exercise dates. For the first test, we select $J=2^{7}$ continuation and early-exercise regions. The results in Table 12 show convergence in $N_{1}$ and $N_{2}$, and the prices for Sets A and B match the results in [13] very well. Convergence for Set C, with $q_{F}=-0.84$, is somewhat slow because of the slowly decreasing Fourier coefficients in this case. The quadrature-COS method [13] gives the reference value 5.3982 .

In a second test we vary the number of continuation and exercise regions. The convergence is quadratic in $J$; see Table 13.

The computation times are significantly longer than for the Bermudan rainbow options under Lévy processes in section 6.2 because efficient matrix-vector multiplication with the FFT algorithm is applicable in only one direction; see section 4 . However, accuracy of four digits can still be obtained within seconds for Sets A and B.

8. Higher-dimensional COS method. The 2D-COS formula can be generalized to higher dimensions easily. In this section we elaborate on the possibilities and difficulties of the higher-dimensional COS method. In section 8.1, we demonstrate an example with three underlying assets.

Suppose we have an $n$-dimensional asset price process, with $N_{1}=N_{2}=\cdots=$ $N_{n}=N$ terms in the series summations. Then, with the methodology that is described in section 3, the computational complexity of pricing a Bermudan option is $O\left(2^{n} N^{n} \log _{2} N\right)$. It is clear that we cannot choose $n$ too large, as then the curse of dimensionality sets in. Besides, the data storage grows exponentially in $n$. For example, in seven dimensions, with $N=100$, the storage of the Fourier coefficients $V\left(t_{m}\right)$ requires $8 \cdot 10^{5}$ GB memory. Dimensions $n \in\{2, \ldots, 5\}$ should, however, still be fine. For higher dimensions, efficient aggregation of states or sparse grid methods 
TABLE 12

Results for Bermudan put option $\left(J=2^{7}\right)$.

(a) Option values, Set A $\left(q_{F}=0.98\right)$.

\begin{tabular}{r|cccc}
\hline$N_{1}$ & \multicolumn{4}{|c}{$N_{2}$} \\
& 40 & 60 & 80 & 100 \\
\hline 40 & 0.517765 & 0.517869 & 0.517894 & 0.517903 \\
60 & 0.517176 & 0.517285 & 0.517311 & 0.517320 \\
80 & 0.517021 & 0.517130 & 0.517156 & 0.517165 \\
100 & 0.517008 & 0.517116 & 0.517143 & 0.517152 \\
\hline
\end{tabular}

(b) Option values, Set B $\left(q_{F}=-0.47\right)$

\begin{tabular}{r|cccc}
\hline$N_{1}$ & \multicolumn{4}{|c}{$N_{2}$} \\
& 40 & 60 & 80 & 100 \\
\hline 40 & 3.20083 & 3.20077 & 3.20071 & 3.20066 \\
60 & 3.19909 & 3.19903 & 3.19897 & 3.19893 \\
80 & 3.19912 & 3.19907 & 3.19901 & 3.19897 \\
100 & 3.19910 & 3.19905 & 3.19899 & 3.19894 \\
\hline
\end{tabular}

(c) Option values, Set C $\left(q_{F}=-0.84\right)$.

(d) CPU time (s).

\begin{tabular}{|c|c|c|c|c|c|c|c|c|c|}
\hline \multirow{2}{*}{$N_{1}$} & \multicolumn{4}{|c|}{$N_{2}$} & \multirow{2}{*}{$N_{1}$} & \multicolumn{4}{|c|}{$N_{2}$} \\
\hline & 40 & 60 & 80 & 100 & & 40 & 60 & 80 & 100 \\
\hline 40 & 5.70464 & 5.57416 & 5.50068 & 5.45386 & 40 & 6.7 & 10.5 & 14.8 & 22.8 \\
\hline 60 & 5.77720 & 5.67101 & 5.61174 & 5.57399 & 60 & 8.8 & 14.0 & 20.2 & 31.2 \\
\hline 80 & 5.75689 & 5.64811 & 5.58898 & 5.55195 & 80 & 11.2 & 17.9 & 25.8 & 42.9 \\
\hline 100 & 5.75808 & 5.64673 & 5.58552 & 5.54697 & 100 & 13.5 & 21.4 & 31.2 & 51.2 \\
\hline
\end{tabular}

TABLE 13

Bermudan option values $\hat{v}\left(t_{0}, \mathbf{x}\right)$, convergence in $J\left(N_{1}=100, N_{2}=100\right)$.

\begin{tabular}{l|ccccc}
\hline $\log _{2} J$ & 3 & 4 & 5 & 6 & 7 \\
\hline Set A & 0.515991 & 0.516784 & 0.517060 & 0.517133 & 0.517152 \\
Set B & 3.186917 & 3.196282 & 3.198380 & 3.198837 & 3.198944 \\
Set C & 5.42431 & 5.51038 & 5.53874 & 5.54523 & 5.54697 \\
CPU time (s) & 3.4 & 6.3 & 12.4 & 25.1 & 51.2 \\
\hline
\end{tabular}

are needed. However, most existing methods for a dimensionality of 10 and higher are based on Monte Carlo methods.

For pricing Bermudan options we need to determine $n$-dimensional continuation and early-exercise boxes. In three-dimensional (3D) space, we get rectangular cuboids. For four or higher dimensions it gets harder to visualize the regions. Special algorithms to determine the regions efficiently, or even adaptively, may be helpful.

The terminal coefficients $V_{k_{1}, k_{2}, \ldots, k_{n}}(T)$ can be approximated by using DCTs; see section 3.2.2. Computation or approximation of the multidimensional coefficients $G_{k_{1}, k_{2}, \ldots, k_{n}}\left(\mathcal{G}^{p}\right)$ can be time consuming or no analytic expression may be available, but we can use the terminal coefficients to approximate them. This approach was explained in section 3.2.2.

8.1. 3D-COS formula. The $3 \mathrm{D}$ version of the COS formula reads as

$$
\begin{aligned}
\hat{v}\left(t_{0}, \mathbf{x}\right):= & \prod_{i=1}^{3} \frac{b_{i}-a_{i}}{2} e^{-r \Delta t} \sum_{k_{1}=0}^{N_{1}-1} \sum_{k_{2}=0}^{\prime N_{2}-1} \sum_{k_{3}=0}^{\prime N_{3}-1} \frac{1}{4} \\
& {\left[F_{k_{1}, k_{2}, k_{3}}^{++}(\mathbf{x})+F_{k_{1}, k_{2}, k_{3}}^{+-}(\mathbf{x})+F_{k_{1}, k_{2}, k_{3}}^{-+}(\mathbf{x})+F_{k_{1}, k_{2}, k_{3}}^{--}(\mathbf{x})\right] V_{k_{1}, k_{2}, k_{3}}(T) . }
\end{aligned}
$$

Copyright $\odot$ by SIAM. Unauthorized reproduction of this article is prohibited. 
The coefficients $F_{k_{1}, k_{2}, k_{3}}^{ \pm \pm}$are given by

$$
\begin{array}{r}
F_{k_{1}, k_{2}, k_{3}}^{ \pm \pm}(\mathbf{x})=\prod_{i=1}^{3} \frac{2}{b_{i}-a_{i}} \operatorname{Re}\left(\varphi\left(\frac{k_{1} \pi}{b_{1}-a_{1}}, \pm \frac{k_{2} \pi}{b_{2}-a_{2}}, \pm \frac{k_{3} \pi}{b_{3}-a_{3}} \mid \mathbf{x}\right)\right. \\
\left.\exp \left(-i k_{1} \pi \frac{a_{1}}{b_{1}-a_{1}} \mp i k_{2} \pi \frac{a_{2}}{b_{2}-a_{2}} \mp i k_{3} \pi \frac{a_{3}}{b_{3}-a_{3}}\right)\right) \\
=\prod_{i=1}^{3} \frac{2}{b_{i}-a_{i}} \operatorname{Re}\left(\varphi_{\text {levy }}\left(\frac{k_{1} \pi}{b_{1}-a_{1}}, \pm \frac{k_{2} \pi}{b_{2}-a_{2}}, \pm \frac{k_{3} \pi}{b_{3}-a_{3}}\right)\right. \\
\left.\exp \left(i k_{1} \pi \frac{x_{1}-a_{1}}{b_{1}-a_{1}} \pm i k_{2} \pi \frac{x_{2}-a_{2}}{b_{2}-a_{2}} \pm i k_{3} \pi \frac{x_{3}-a_{3}}{b_{3}-a_{3}}\right)\right),
\end{array}
$$

where the last equality holds for Lévy processes, with $\varphi_{\text {levy }}\left(u_{1}, u_{2}, u_{3}\right):=\varphi\left(u_{1}, u_{2}\right.$, $\left.u_{3} \mid 0,0,0\right)$.

We test the 3D-COS formula by pricing European basket call options, with parameters from Set IV. The results in [21] are used as reference prices. For the coefficients $V_{k_{1}, k_{2}, k_{3}}(T)$ we use DCTs to approximate them. The results are presented in Table 14. They show that the multidimensional-COS formula is very accurate. The CPU time for $N_{1}=40$ and $Q=200$ is less than 2 seconds.

\begin{tabular}{|c|c|c|c|c|c|c|c|c|c|}
\hline \multirow[b]{2}{*}{$Q$} & \multirow{2}{*}{$N_{1}$} & \multicolumn{4}{|c|}{ Geometric basket } & \multicolumn{4}{|c|}{ Arithmetic basket } \\
\hline & & 10 & 20 & 40 & 80 & 10 & 20 & 40 & 80 \\
\hline & 50 & 1.78 & $3.12 \mathrm{e}-2$ & $4.03 \mathrm{e}-3$ & $\mathrm{n} / \mathrm{a}$ & $2.11 \mathrm{e}+1$ & $4.59 \mathrm{e}-1$ & $6.94 \mathrm{e}-3$ & $\mathrm{n} / \mathrm{a}$ \\
\hline & 100 & 1.79 & $4.53 \mathrm{e}-2$ & $7.92 \mathrm{e}-3$ & $7.91 \mathrm{e}-3$ & $2.14 \mathrm{e}+1$ & $4.80 \mathrm{e}-1$ & $9.82 \mathrm{e}-5$ & $7.82 \mathrm{e}-5$ \\
\hline & 200 & 1.81 & $3.68 \mathrm{e}-2$ & $9.88 \mathrm{e}-4$ & $9.89 \mathrm{e}-4$ & $2.15 \mathrm{e}+1$ & $4.86 \mathrm{e}-1$ & $<5.0 \mathrm{e}-5$ & $2.13 \mathrm{e}-5$ \\
\hline & 400 & 1.81 & $3.77 \mathrm{e}-2$ & $2.46 \mathrm{e}-4$ & $2.47 \mathrm{e}-4$ & $2.15 \mathrm{e}+1$ & $4.87 \mathrm{e}-1$ & $<5.0 \mathrm{e}-5$ & $<5.0 \mathrm{e}-5$ \\
\hline
\end{tabular}

TABLE 14

Absolute error for the geometric basket call option, $v\left(t_{0}, \mathbf{x}_{0}\right)=11.9791$, and the arithmetic basket call option, $v\left(t_{0}, \mathbf{x}_{0}\right)=13.2449\left(N_{1}=N_{2}=N_{3}\right)$.

9. Conclusion. The 1D-COS method for pricing European and Bermudan options was developed in $[11,12]$. The method is based on Fourier cosine expansions, and the series coefficients of the value function are recovered backwards in time. In this paper we presented the generalization of the COS method to higher dimensions. The recursive algorithm can be applied to, for example, pricing rainbow options but also to pricing under the Heston stochastic volatility model.

The 2D-COS method for valuation of Bermudan options is based on the dynamic programming principle and 2D Fourier cosine series expansions. For smooth density functions, the resulting method converges exponentially in $N_{1}$ and $N_{2}$, the number of terms in the series summations. Otherwise we achieve algebraic convergence, as in the 1D case. For multidimensional stochastic processes in the class of Lévy processes, we can apply efficient matrix-vector multiplication using the FFT algorithm. The Heston model is not in the Lévy class, and the FFT algorithm will be applicable in only one dimension.

We performed extensive numerical experiments with European and Bermudan options. The algorithm for pricing two-color rainbow options can be used for various payoffs and performs highly satisfactorily. The asset prices in the tests are modeled by GBMs or correlated jump-diffusions, but the method can be applied to a broad 
class of multidimensional asset price processes for which the characteristic function is available.

The density function of the Heston dynamics may be nonsmooth, especially near zero variance if the Feller condition is not satisfied, and peaked densities give rise to slow algebraic convergence. However, for a wide range of parameter values, the 2D-COS method achieves accurate Bermudan put prices.

\section{Appendix A. FFT algorithm.}

THEOREM A.1 (efficient computation of $\hat{C}$ and $\mathcal{A}^{q}$ ). The matrix-vector product $M^{+}\left(u_{1}, u_{2}, a, b\right) \mathbf{v}$ and $M^{-}\left(u_{1}, u_{2}, a, b\right) \mathbf{v}$ can be computed in $O\left(N \log _{2} N\right)$ operations with the help of the FFT algorithm, with $N$ the size of square-matrices $M^{+}$and $M^{-}$.

The key insights of this efficient computation are the equalities

$$
\begin{aligned}
& M_{k, j}^{+}\left(z_{1}, z_{2}, a, b\right)=-\frac{i}{\pi}\left(M_{k, j}^{+c}\left(z_{1}, z_{2}, a, b\right)+M_{k, j}^{+s}\left(z_{1}, z_{2}, a, b\right)\right), \\
& M_{k, j}^{-}\left(z_{1}, z_{2}, a, b\right)=-\frac{i}{\pi}\left(M_{k, j}^{-c}\left(z_{1}, z_{2}, a, b\right)+M_{k, j}^{-s}\left(z_{1}, z_{2}, a, b\right)\right),
\end{aligned}
$$

where

$$
\begin{aligned}
& M_{k, j}^{ \pm c}\left(z_{1}, z_{2}, a, b\right)=\frac{\exp \left(i( \pm j+k) \frac{\left(z_{2}-a\right) \pi}{b-a}\right)-\exp \left(i( \pm j+k) \frac{\left(z_{1}-a\right) \pi}{b-a}\right)}{ \pm j+k}, \\
& M_{k, j}^{ \pm s}\left(z_{1}, z_{2}, a, b\right)=\frac{\exp \left(i( \pm j-k) \frac{\left(z_{2}-a\right) \pi}{b-a}\right)-\exp \left(i( \pm j-k) \frac{\left(z_{1}-a\right) \pi}{b-a}\right)}{ \pm j-k},
\end{aligned}
$$

with special cases

$$
\begin{array}{ll}
M_{k, j}^{+c}\left(z_{1}, z_{2}, a, b\right)=\frac{\left(z_{2}-z_{1}\right) \pi i}{b-a} & \text { for } k=j=0, \\
M_{k, j}^{-s}\left(z_{1}, z_{2}, a, b\right)=\frac{\left(z_{2}-z_{1}\right) \pi i}{b-a} & \text { for } k=j=0, \\
M_{k, j}^{-c}\left(z_{1}, z_{2}, a, b\right)=\frac{\left(z_{2}-z_{1}\right) \pi i}{b-a} & \text { for } k=j, \\
M_{k, j}^{+s}\left(z_{1}, z_{2}, a, b\right)=\frac{\left(z_{2}-z_{1}\right) \pi i}{b-a} & \text { for } k=j .
\end{array}
$$

The matrices $M^{+c}$ and $M^{-s}$ are Hankel matrices and $M^{+s}$ and $M^{-c}$ are Toeplitz matrices. These special structures make the calculation of the matrix-vector products efficient using FFT algorithms, as described in [12, 4].

Acknowledgments. The authors wish to thank Prof. Dr. F. H. J. Redig and Dr. J. A. M. van der Weide for helpful suggestions and discussions.

\section{REFERENCES}

[1] H. Albrecher, P. Mayer, W. Schoutens, and J. Tistaert, The little Heston trap, Wilmott Magazine, January (2007), pp. 83-92.

[2] L. Andersen And M. Broadie, Primal-dual simulation algorithm for pricing multidimensional American options, Management Sci., 50 (2004), pp. 1222-1234.

[3] S. J. Berridge and J. M. Schumacher, Pricing high-dimensional American options using local consistency conditions, in Numerical Methods for Finance, J. Miller, D. Edelman, and J. Appleby, eds., Chapman \& Hall/CRC, London, 2007, pp. 13-52.

Copyright $@$ by SIAM. Unauthorized reproduction of this article is prohibited. 
[4] J. P. Boyd, Chebyshev and Fourier Spectral Methods, Dover, Mineola, NY, 2001.

[5] P. P. Boyle, A lattice framework for option pricing with two state variables, J. Financ. Quant. Anal., 23 (1988), pp. 1-12.

[6] P. P. Boyle, J. Evnine, And S. GibBs, Numerical evaluation of multivariate contingent claims, Rev. Financ. Stud., 2 (1989), pp. 241-250.

[7] M. Broadie and J. Detemple, The valuation of American options on multiple assets, Math. Finance, 7 (1997), pp. 241-286.

[8] S. S. Clift and P. A. Forsyth, Numerical solution of two asset jump diffusion models for options valuation, Appl. Numer. Math., 58 (2008), pp. 743-782.

[9] D. Duffie, J. Pan, And K. J. Singleton, Transform analysis and asset pricing for affine jump-diffusions, Econometrica, 68 (2000), pp. 1343-1376.

[10] D. Dufresne, Bessel processes and Asian options, in Numerical Methods in Finance, H. BenAmeur and M. Breton, eds., Springer-Verlag, New York, 2005, pp. 35-57.

[11] F. Fang And C. W. Oosterlee, A novel pricing method for European options based on Fourier-cosine series expansions, SIAM J. Sci. Comput., 31 (2008), pp. 826-848.

[12] F. Fang And C. W. Oosterlee, Pricing early-exercise and discrete barrier options by Fouriercosine series expansions, Numer. Math., 114 (2009), pp. 27-62.

[13] F. Fang and C. W. Oosterlee, A Fourier-based valuation method for Bermudan and barrier options under Heston's model, SIAM J. Financial Math., 2 (2011), pp. 439-463.

[14] S. L. Heston, A closed-form solution for options with stochastic volatility with applications to bond and currency options, Rev. Financ. Stud., 6 (1993), pp. 327-343.

[15] S.-F. Huang And M. Guo, Valuation of multidimensional bermudan options, in Applied Quantitative Finance, H. K. Wolfgang, N. Hautsch, and L. Overbeck, eds., Springer-Verlag, Berlin, Heidelberg, 2008, pp. 295-309.

[16] S. Jain And C. W. Oosterlee, Pricing high-dimensional Bermudan options using the stochastic grid method, Int. J. Comput. Math., 89 (2012), pp. 1186-1211.

[17] A. Janek, T. Kluge, R. Weron, And U. Wystup, FX smile in the Heston model, in Statistical Tools for Finance and Insurance, P. Čížek, W. K. Härdle, and R. Weron, eds., SpringerVerlag, Heidelberg, 2011, pp. 133-162.

[18] M. Jeanblanc, M. Yor, and M. Chesney, Mathematical Methods for Financial Markets, Springer Finance, Springer-Verlag, London, 2009.

[19] C. Kahl and P. JÄCKel, Not-so-complex logarithms in the Heston model, Wilmott Magazine, September (2005), pp. 94-103

[20] S. Kehtari, private communication, ETH Zürich, Zürich, Switzerland.

[21] C. C. W. LeEntvaAR And C. W. OOsterlee, On coordinate transformation and grid stretching for sparse grid pricing of basket options, J. Comput. Appl. Math., 222 (2008), pp. 193-209.

[22] C. C. W. LeentvaAR AND C. W. Oosterlee, Multi-asset option pricing using a parallel Fourier-based technique, J. Comput. Finance, 12 (2008), pp. 1-26.

[23] R. Lord And C. Kahl, Why the Rotation Count Algorithm Works, Tinbergen Institute Discussion Paper 2006-065/2, Tinbergen Institute, Rotterdam, The Netherlands, 2006.

[24] R. Lord And C. KAhl, Complex logarithms in Heston-like models, Math. Finance, 20 (2010), pp. 671-694.

[25] S. Pagliarani, A. Pascucci, and C. Riga, Expansion Formulae for Local Lévy Models, http://ssrn.com/abstract=1937149 (2011).

[26] T. T. Soong, Random Differential Equations in Science and Engineering, Academic Press, New York, London, 1973.

[27] R. M. Stulz, Options on the minimum or the maximum of two risky assets: Analysis and applications, J. Financ. Econ., 10 (1982), pp. 161-185.

[28] B. Zhang, L. A. Grzelak, and C. W. Oosterlee, Efficient pricing of commodity options with early-exercise under the Ornstein-Uhlenbeck process, Appl. Numer. Math., 62 (2012), pp. 91-111.

Copyright (C) by SIAM. Unauthorized reproduction of this article is prohibited. 